\title{
Répteis do Mato Grosso do Sul, Brasil
}

\author{
Vanda Lúcia Ferreira', Juliana de Souza Terra², Liliana Piatti', Milena Delatorre², Christine \\ Strüssmann ${ }^{3}$, Arlindo F. Béda ${ }^{4}$, Ricardo A. Kawashita-Ribeiro ${ }^{5}$, Paulo Landgref-Filho ${ }^{4}$, Camila Aoki ${ }^{4}$, Zilca \\ Campos ${ }^{6}$, Franco L. Souza ${ }^{7}$, Robson W. Ávila ${ }^{7}, S^{2}$, \\ Paula Helena Santa Rita $^{10}$ \& Nelson R. Albuquerque ${ }^{11}$
}

\author{
1. Universidade Federal de Mato Grosso do Sul, Centro de Ciências Biológicas e da Saúde, Campo Grande, MS. (vandalferreira@gmail.com) \\ 2. Universidade Federal de Mato Grosso do Sul, Programa de Pós-Graduação em Ecologia e Conservação, Campo Grande, MS. \\ 3. Universidade Federal de Mato Grosso, Faculdade de Agronomia, Medicina Veterinária e Zootecnia, Departamento de Ciências Básicas e Produção Animal \\ Cuiabá, MT. \\ 4. Universidade Federal de Mato Grosso do Sul, Campus Universitário de Aquidauana - CPAQ, Departamento de Biociências, Aquidauana, MS. \\ 5. Universidade Federal de Mato Grosso, Instituto de Biociências, Programa de Pós-Graduação em Ecologia e Conservação da Biodiversidade, Cuiabá, MT. \\ 6. Empresa Brasileira de Pesquisa Agropecuária, Embrapa Pantanal, Corumbá, MS \\ 7. Universidade Regional do Cariri, Departamento de Ciências Físicas e Biológicas, Crato, CE. \\ 8. Universidade Federal de Mato Grosso do Sul, Centro de Ciências Biológicas e da Saúde, Programa de Pós-Graduação em Biologia Animal, \\ Campo Grande, MS. \\ 9. Universidade Federal de Mato Grosso do Sul, Centro de Ciências Biológicas e da Saúde, Curso de Ciências Biológicas, Campo Grande, MS \\ 10. Universidade Católica Dom Bosco, Biotério, Campo Grande, MS. \\ 11. Universidade Federal de Mato Grosso do Sul, Campus do Pantanal, Corumbá, MS.
}

Recebido 8 dezembro 2016

Aceito 6 fevereiro 2017

DOI: $10.1590 / 1678-4766 e 2017153$

ABSTRACT. Reptiles of Mato Grosso do Sul, Brazil. Data is presented on richness and composition of reptiles from the state of Mato Grosso do Sul (MS), based on reviews of publications, examination of scientific collections and unpublished field data provided by researchers. We recorded 188 species distributed in eight species of Testudines, three Crocodylia and 177 Squamata (16 amphisbaenians, 48 lizards and 113 snakes). Thirteen species have restricted distributions within the state, 13 are restricted to the state and nine are in the process of being described. The diversity of the MS reptiles, even if underestimated, accounts for $25 \%$ of the species known to occur in Brazil. The plateau presents 176 species, of which $30 \%$ are restricted to this location, while 131 species ( $6 \%$ exclusive) occur in the floodplain. The Cerrado is the richest domain in species, although it is the region with the highest sampling gap in the state. The most well-known section is the Midwest MS, which includes the Pantanal. About $40 \%$ of the species of reptiles of MS are widely distributed, occurring in at least five of the seven Brazilian domains. The reptiles of MS are more similar to those of the Chaco, probably because the chacoan fauna is very similar to that of the Pantanal, which includes better inventoried portions of MS. Although the Cerrado is the predominant domain in the state, the fact of being poorly sampled justifies the lower similarity of reptiles from this domain with those of MS, when compared with those of Chaco. Overall, the MS reptile fauna shows greater similarity to that found along the diagonal of open formations of South America. Only $18 \%$ of species were assessed by IUCN, which reinforces the need for an assessment of the conservation status of MS reptile species. The north, south and east of the state have not being adequately sampled, which is of concern since these sections have seen significant anthropogenic alteration. For this reason, these areas, especially the north - including the headwaters of rivers which supply the floodplain - are indicated as priorities for the completion of inventories of reptiles and creation of areas to protect them.

KEYWORDS. Biodiversity, Cerrado, Chaco, herpetofauna, Pantanal, Biota-MS Program.

RESUMO. Informações sobre riqueza e composição de répteis pela primeira vez são apresentadas para o Mato Grosso do Sul (MS). A compilação de dados foi baseada em publicações, coleções científicas e dados disponibilizados por pesquisadores. Foram registradas 188 espécies distribuídas em oito espécies de Testudines, três Crocodylia e 177 Squamata (16 anfisbenas, 48 sáurios e 113 serpentes). Treze espécies, dentro do estado, possuem distribuição restrita, 13 não são encontradas em outros estados brasileiros e nove estão em processo de descrição. A riqueza de répteis do MS, mesmo que subestimada, corresponde a $25 \%$ das espécies conhecidas no Brasil. No planalto estão presentes 176 espécies, das quais $30 \%$ foram registradas somente neste local, enquanto que na planície de inundação ocorrem 131 espécies (6\% exclusivas). O Cerrado é o domínio mais rico em espécies, ainda que esteja na região com maior lacuna de amostragem do estado. A porção mais conhecida é o centro-oeste do MS, que inclui o Pantanal. Cerca de $40 \%$ das espécies de répteis deste estudo tem ampla distribuição, ocorrendo em pelo menos cinco dos sete domínios brasileiros. Os répteis do estado são mais semelhantes àqueles do Chaco, provavelmente porque a fauna chaquenha é muito similar a do Pantanal, que contempla porções mais bem inventariadas do MS. Ainda que o Cerrado seja o domínio predominante no estado, o fato de ser pouco amostrado justifica a menor similaridade da sua fauna de répteis com o MS, quando comparado àquela do Chaco. De modo geral, a fauna estadual de répteis é mais parecida com aquela encontrada ao longo da diagonal de formações abertas da América do Sul. Apenas 18\% das espécies foram avaliadas pela IUCN, o que reforça a necessidade de uma avaliação do estado de conservação das espécies do MS. O norte, sul e leste do estado não foram minimamente amostrados, o que é preocupante visto que são regiões comprometidas pelo intensivo uso da terra. Por essa razão, essas áreas, e em especial o norte, por incluir as cabeceiras dos rios que abastecem a planície de inundação, são indicadas como prioritárias para a realização de inventários de répteis e criação de UCs.

PALAVRAS-CHAVES. Biodiversidade, Cerrado, Chaco, herpetofauna, Pantanal, Programa Biota-MS. 
A diversidade de répteis do estado do Mato Grosso do Sul (MS) é pouco conhecida, sendo que os registros de espécies são ocasionais ou restritos a algumas regiões (ver FerReira et al., 2005; KaWASHITA-Ribeiro, 2007; StrüsSMANn et al., 2007, 2011; UetanABaro et al., 2007; Souza et al., 2010). Desde as primeiras estimativas de riqueza, até hoje, a maior quantidade de informações sobre os répteis do MS são proveniente de estudos focados na porção brasileira da bacia hidrográfica do Alto Paraguai (BAP), que inclui o MS e o Mato Grosso (MT) (e.g., BrAsIL, 1997; SWARTZ, 2000; AlHO et al., 2001; JUNK et al., 2006; STRÜSSMANN et al., 2007, 2011). No MS, a BAP abrange regiões da margem direta e esquerda do rio Paraguai e seus afluentes da margem esquerda, o que inclui o Pantanal e Chaco.

A atual avaliação de riqueza desses vertebrados na porção brasileira da BAP totaliza cerca de 200 espécies (STRÜSSMANN et al., 2011), número que tem sido incrementado com recentes ampliações de distribuição (e.g., ARrudA et al., 2011; Albuquerque \& Lema, 2012; Barreto et al., 2012; SugAi et al., 2012). Informações disponíveis para a BAP e outras regiões do MS (BrasIL, 1997; StRÜSSMANN et al., 2000; WANG et al., 2004, 2005; UetanABARo et al., 2007; Souza et al., 2010) indicam haver substituição de espécies de répteis entre as regiões. E ainda, ao menos para as taxocenoses de serpentes do estado (ver STRÜSSMANN et al., 2007; KaWASHITA-RIBEIRo, 2007; Ferrão, 2011) é possível sugerir que são mais similares àquelas inseridas na diagonal de formações abertas da América do Sul (Caatinga, Cerrado e Chaco), como preconizado por VANZOLINI (1963) e ColLi (2005).

O estado situa-se na porção central da diagonal de formações abertas, onde ocorrem áreas de contato com os domínios do Pantanal, Chaco e Cerrado (Uetanabaro et al., 2007). Os domínios do Pantanal e do Chaco estão presentes em uma das duas distintas macrorregiões resultantes de variações no relevo - a planície de inundação (PRADo et al., 1992; LouRIVAL et al., 2000). Ambos situados na porção oeste do MS, o Pantanal ocupa aproximadamente $20 \%$ e o Chaco $3 \%$ do território estadual; este último é representado no país unicamente no município de Porto Murtinho (HARRIs et al., 2005a; SouZA et al., 2010). A outra macrorregião marcante no MS é o planalto, com áreas mais elevadas (de $200 \mathrm{~m}$ a pouco mais de $1000 \mathrm{~m}$ de altitude) e não inundáveis (LouRIVAL et al., 2000). Localizado no leste do estado, o planalto abrange o domínio do Cerrado, que corresponde à maior parte do território estadual ( $60 \%)$, e da Mata Atlântica ( $14 \%$ ) que ocorre no sul (IBGE, 2011).

O Cerrado brasileiro tem sido contemplado com maior número de estudos, mas a fauna de répteis em alguns habitats e áreas não foi adequadamente amostrada (Colli et al., 2002), incluindo a porção de Cerrado do MS (LouRIVAL et al., 2000; STRÜSSMANN et al., 2000, 2007). Apesar do aumento no número de estudos no estado, em particular no Pantanal, que é foco de ações conservacionistas, os padrões de riqueza, abundância e composição de répteis ainda permanecem praticamente desconhecidos (STRÜSSMANN et al., 2007, 2011).
Ao longo da última década, cerca de $70 \%$ da vegetação nativa do MS foi suprimida por atividades antrópicas, principalmente relacionadas à agropecuária e mineração (ver HARRIs et al., 2005a; FundAÇÃo CÂNDIDO RONDON, 2007). Nos últimos anos, áreas de pastagens têm sido substituídas por lavouras de cana, soja e eucalipto, principalmente nas áreas de Cerrado (Klink \& Moreira, 2002; Almeida, 2011; PIAU, 2012). Ainda assim, o estado destaca-se pela heterogeneidade de fitofisionomias e por possuir áreas de declarada importância biológica reconhecidas nacional e internacionalmente (MMA, 2002, 2007a,b).

Porções do território do MS foram incluídas há 25 anos como "Patrimônio Nacional" na Constituição Brasileira. Posteriormente foram consideradas como Patrimônio Natural da Humanidade pela Organização das Nações Unidas para a Educação, Ciência e Cultura (UNESCO) e inseridas como uma das Áreas Prioritárias para a Conservação, Utilização Sustentável e Repartição de Benefícios da Biodiversidade Brasileira (MMA 2002, 2007a,b). Algumas áreas do estado foram também inseridas na Reserva da Biosfera do Pantanal (RBMA, 2007), além de constituírem um dos sítios RAMSAR (a convenção sobre zonas úmidas de importância internacional; http://www.ramsar.org/wetland/ brazil) e serem indicadas para integrar o Corredor Ecológico Cerrado-Pantanal (Lourival et al., 2000).

Frente ao aumento de dados e à necessidade de subsidiar o conhecimento de história natural e ações de conservação das espécies do MS, o estudo apresenta a riqueza e composição de répteis do estado, além de indicar lacunas de conhecimento.

\section{MATERIAL E MÉTODOS}

Para a elaboração da lista de espécies foram compilados dados da literatura científica dados não publicados disponibilizados por pesquisadores, e registros de coleções científicas. As coleções consultadas (com suas respectivas acronímias) foram: Coleção de Vertebrados do Pantanal (HCPAP), Embrapa Pantanal, Corumbá, MS; Coleção Herpetológica “Alphonse Richard Hoge", Instituto Butantan (IBSP), São Paulo, SP; Coleção Herpetológica da Universidade de Brasília (CHUNB), DF; Coleção Herpetológica do Biotério da Universidade Católica Dom Bosco (BIOTH), Campo Grande, MS; Coleção Zoológica de Referência da Universidade Federal de Mato Grosso do Sul (ZUFMS), Campo Grande, MS; Coleção Zoológica de Referência do Campus de Corumbá, Seção de Herpetologia da Universidade Federal de Mato Grosso do Sul (CEUCH), Campo Grande, MS; Coleção Zoológica de Vertebrados da Universidade Federal de Mato Grosso (UFMT), Cuiabá, MT; Museu de História Natural Capão da Imbuia (MHNCI), Curitiba, PR; Museu de Zoologia da Universidade de São Paulo (MZUSP), SP; Museu de Zoologia da Universidade Estadual de Londrina (MZUEL), PR.

Foram extraídas, de publicações, referências a material testemunho em algumas coleções não consultadas, entre elas: Fundación Miguel Lillo (FML), Tucumán, Argentina; 
Museo de La Plata (MLP), Argentina; Museo Regionale di Scienze Naturali di Torino (MZUT), Itália; Museu de Ciências e Tecnologia da Pontifícia Universidade Católica do Rio Grande do Sul (MCP), Porto Alegre, RS; Museu Nacional da Universidade Federal do Rio de Janeiro (MNRJ), RJ; University of Michigan Museum of Zoology (UMMZ), Ann Arbor, Michigan, USA. A Coleção Herpetológica Arlindo de Figueiredo Béda (CHAFB) foi incorporada à ZUFMS em 2010, de forma que o material testemunho citado como CHAFB em publicações foi aqui considerado como depositado na ZUFMS.

Ainda, foram utilizadas informações confiáveis (segundo julgamento dos autores) que constam em planos de manejo de Reservas Particulares do Patrimônio Natural (RPPNs), diagnósticos ambientais, estudos de impacto ambiental (EIA) e monitoramentos ambientais de empreendimentos instalados no estado. A maior parte destes documentos está sob a custódia do Instituto do Meio Ambiente de Mato Grosso do Sul (IMASUL) e do Instituto Brasileiro do Meio Ambiente e dos Recursos Naturais Renováveis (IBAMA).

Registros de coleções ou de literatura cuja procedência e/ou identificação foram questionados pelos autores, que não puderam ser confirmados por material testemunho, foram desconsiderados. No caso de exemplares cuja identificação da espécie não foi possível, o registro foi considerado apenas para as categorias de gênero e família (e.g., Amphisbaena sp., Phalotris gr. tricolor). Espécie que sabidamente é nova para a ciência, mas que ainda não foi formalmente descrita, foi indicada como "sp. nov." (e.g., Ameiva sp. nov.).

As coordenadas geográficas de cada registro foram obtidas em campo através do sistema global de posicionamento (GPS; Datum: WGS84) e/ou a partir da localização aproximada com auxílio de imagens do software Google Earth ${ }^{\mathrm{TM}}$. Quando somente o município foi informado, usou-se a coordenada centroide do município, calculada por meio de mapas da malha municipal brasileira fornecidos pelo Instituto Brasileiro de Geografia e Estatística (IBGE), com os pacotes Maptools (LewIN-KoH et al., 2009) e Rgeos (BIVAND \& RuNDEL, 2012), ambos utilizados no software R (R CORE TEAm, 2012).

As espécies foram relacionadas às duas macrorregiões fisiográficas consideradas: "planície de inundação" (PI) e "planalto" (PL). Áreas montanhosas, mesmo que nos limites da planície de inundação, foram consideradas como planalto (e.g., morro Santa Cruz, Urucum, Rabicho, Bocaina, Serra do Amolar, Serra de Maracaju, Serra da Bodoquena) (ver BRASIL, 1997).

Adicionalmente, foi produzido o mapa de lacunas de inventários de répteis no MS através do método de interpolação circular neighborhood (CRESSIE, 1991), tendo sido analisados os registros dos pontos de amostragens com auxílio do Programa DIVA-GIS (Humans et al., 2001). Dessa forma, foi obtido um mapa no formato rasterizado, o qual indica as regiões mais conhecidas e áreas que apresentam lacunas de informações sobre a fauna de répteis do estado. A partir das coordenadas dos locais amostrados, foi gerado um mapa que representa a distribuição desses pontos nos principais tipos de vegetação (IBGE, 2004) (http://www.ibge. gov.br/home/geociencias/default_prod.shtm), nas unidades de conservação (UCs) de proteção integral e RPPNs do estado criadas até 2011 (http://www.ibama.gov.br/zoneamentoambiental).

A composição de répteis reportada para o MS, baseada na incidência de espécies, foi comparada àquelas encontradas nos domínios fitogeográficos do Brasil (Caatinga, Cerrado, Campos Sulinos, Floresta Amazônica e Mata Atlântica) e no Chaco, domínio também presente nos países vizinhos (Argentina e Paraguai). O Pantanal não foi incluído nesta análise, pois os poucos trabalhos nesse domínio incluem dados do estado, já que $80 \%$ deste está no território do MS. As espécies marinhas, insulares e taxonomicamente indefinidas encontradas na literatura, bem como espécies do estado sob revisão, não foram consideradas. Utilizou-se o índice de dissimilaridade de Sorensen para fazer a análise de agrupamento (UPGMA), a qual foi realizada no programa PAST (HAMMER et al., 2001).

Para conhecer o estado de conservação dos répteis do MS foi consultada a Lista Brasileira de Animais Ameaçados de Extinção (MMA, 2003; ICMBio, 2015) e a Lista Vermelha da União Internacional para a Conservação da Natureza e dos Recursos Naturais (IUCN, 2012). A nomenclatura das espécies e a classificação utilizada seguem BÉRNILS \& Costa (2012), CostA \& BÉRnils (2014), complementada por CABRERA (2012) no que diz respeito a Cnemidophorus. E para quantificar e discriminar os grupos de pesquisa do estado que envolvem estudos com répteis, foi utilizada a base de dados do Conselho Nacional de Desenvolvimento Científico e Tecnológico - CNPq (www.cnpq.br).

\section{RESULTADO E DISCUSSÃO}

Dentre os 4.749 registros de répteis obtidos para o MS, foram verificadas 188 espécies (Tab. I). Dessas, oito são de Testudines, três de Crocodylia e 177 de Squamata, sendo 16 espécies de Amphisbaenia, 48 de Sauria e 113 de Serpentes. Pouco mais de $8 \%$ dos registros não puderam ser considerados em nível de espécie, pois pertencem a táxons atualmente sob revisão taxonômica. Aproximadamente $60 \%$ de todos os registros foram obtidos de coleções científicas, as quais contemplam praticamente todas as espécies do MS $(95,8 \%)$ (Tab. I). Dentre as espécies, 78,2\% apresentam material testemunho em coleções no estado (ZUFMS, CEUCH, BIOTH, HCPAP).

Apenas 18\% das espécies de répteis do MS foram avaliadas pela IUCN (2012), das quais seis estão na categoria "vulnerável" e 28 na categoria "menos preocupante" (Tab. I). Nenhuma das espécies de répteis do estado consta na lista oficial de animais ameaçados do Brasil em MMA (2003), mas a serpente Phalotris multipunctatus é indicada na categoria "em perigo" (EN) em ICMBio (2015).

A compilação de dados sobre a fauna de répteis do MS forneceu informações inéditas para a herpetofauna brasileira, mesmo que não tenham sido feitas buscas exaustivas em 
Tab. I. Répteis do estado de Mato Grosso do Sul, Brasil. Ocorrência nas macrorregiões, presença nos domínios de acordo com este estudo e literatura, representatividade em coleções científicas consultadas e atual estado de conservação (segundo IUCN, 2012). MR, Macroregiões: PI, planície de inundação; PL, planalto; CA, Caatinga; CE, Cerrado; CH, Chaco; CS, Campos Sulinos e FA, Floresta Amazônica; MA, Mata Atlântica; PA, Pantanal. CZ: material testemunho depositado em coleções zoológicas (X, coleções nacionais e internacionais - ver em materiais e métodos; *, material depositado em coleções do MS); **, Apostolepis goiasensis foi registrada após a finalização do artigo; EC, estado de conservação (LC, menos preocupante; VU, vulnerável); ${ }^{+}$, espécie introduzida no estado. A autoria do táxon com mais de dois autores foi representada pelo primeiro autor seguido de "et al." para de condensar as informações.

\begin{tabular}{|c|c|c|c|c|c|}
\hline \multirow{2}{*}{ Táxon (número total de espécies) } & \multicolumn{2}{|c|}{ MR } & \multirow{2}{*}{ Domínios } & \multirow{2}{*}{$\mathrm{CZ}$} & \multirow{2}{*}{$\mathrm{EC}$} \\
\hline & PI & PL & & & \\
\hline \multicolumn{6}{|l|}{ TESTUDINES - CRYPTODIRA (4) } \\
\hline \multicolumn{6}{|l|}{ Kinosternidae (1) } \\
\hline Kinosternon scorpioides (Linnaeus, 1766) & & $\mathrm{X}$ & $\mathrm{CA}, \mathrm{CE}, \mathrm{CH}, \mathrm{FA}, \mathrm{PA}$ & & \\
\hline \multicolumn{6}{|l|}{ Emydidae (1) } \\
\hline Trachemys scripta (Schoepff, 1792)+ & & $\mathrm{X}$ & CE, MA & & $\mathrm{LC}$ \\
\hline \multicolumn{6}{|l|}{ Testudinidae (2) } \\
\hline Chelonoidis carbonarius (Spix, 1824) & $\mathrm{X}$ & $\mathrm{X}$ & $\mathrm{CA}, \mathrm{CE}, \mathrm{CH}, \mathrm{FA}, \mathrm{MA}, \mathrm{PA}$ & $\mathrm{X}^{*}$ & \\
\hline Chelonoidis denticulatus (Linnaeus, 1766) & & $\mathrm{X}$ & CE, FA, MA & $\mathrm{X}$ & VU \\
\hline \multicolumn{6}{|l|}{ TESTUDINES - PLEURODIRA (4) } \\
\hline \multicolumn{6}{|l|}{ Chelidae (3) } \\
\hline Acanthochelys macrocephala Rhodin et al., 1984 & $\mathrm{X}$ & $\mathrm{X}$ & $\mathrm{CH}, \mathrm{PA}$ & $\mathrm{X}^{*}$ & VU \\
\hline Mesoclemmys vanderhaegei (Bour, 1973) & & $\mathrm{X}$ & $\mathrm{CE}, \mathrm{CH}, \mathrm{MA}$ & $\mathrm{X}^{*}$ & VU \\
\hline Phrynops geoffroanus (Schweigger, 1812) & $\mathrm{X}$ & $\mathrm{X}$ & $\mathrm{CA}, \mathrm{CE}, \mathrm{CH}, \mathrm{MA}, \mathrm{PA}$ & $\mathrm{X}$ & \\
\hline \multicolumn{6}{|l|}{ Podocnemididae (1) } \\
\hline Podocnemis unifilis Troschel, $1848+$ & $\mathrm{X}$ & & CE, FA, PA & $\mathrm{X}^{*}$ & VU \\
\hline Subtotais: & 4 & 7 & & $6(4 *)$ & \\
\hline \multicolumn{6}{|l|}{ CROCODYLIA (3) } \\
\hline \multicolumn{6}{|l|}{ Alligatoridae (3) } \\
\hline Caiman latirostris (Daudin, 1801) & $\mathrm{X}$ & $\mathrm{X}$ & $\mathrm{CA}, \mathrm{CE}, \mathrm{CH}, \mathrm{CS}, \mathrm{MA}, \mathrm{PA}$ & $\mathrm{X}$ & $\mathrm{LC}$ \\
\hline Caiman yacare (Daudin, 1801) & $\mathrm{X}$ & $\mathrm{X}$ & $\mathrm{CE}, \mathrm{CH}, \mathrm{MA}, \mathrm{PA}$ & $\mathrm{X}^{*}$ & LC \\
\hline Paleosuchus palpebrosus (Cuvier, 1807) & $\mathrm{X}$ & $\mathrm{X}$ & $\mathrm{CA}, \mathrm{CE}, \mathrm{CH}, \mathrm{FA}, \mathrm{MA}, \mathrm{PA}$ & $\mathrm{X}^{*}$ & LC \\
\hline Subtotais: & 3 & 3 & & $3(2 *)$ & \\
\hline \multicolumn{6}{|l|}{ SQUAMATA - SAURIA (48) } \\
\hline Gekkonidae (2) & & & & & \\
\hline Hemidactylus mabouia (Moreau de Jonnès, 1818)+ & $\mathrm{X}$ & $\mathrm{X}$ & $\mathrm{CA}, \mathrm{CE}, \mathrm{CH}, \mathrm{CS}, \mathrm{FA}, \mathrm{MA}, \mathrm{PA}$ & $X^{*}$ & $\mathrm{LC}$ \\
\hline Lygodactylus wetzeli (Smith, Martin \& Swain, 1977) & $\mathrm{X}$ & $\mathrm{X}$ & $\mathrm{CE}, \mathrm{CH}, \mathrm{PA}$ & $\mathrm{X}^{*}$ & \\
\hline Phyllodactylidae (1) & & & & & \\
\hline Phyllopezus pollicaris (Spix, 1825) & $X$ & $\mathrm{X}$ & $\mathrm{CA}, \mathrm{CE}, \mathrm{CH}, \mathrm{MA}, \mathrm{PA}$ & $\mathrm{X}^{*}$ & \\
\hline Sphaerodactylidae (4) & & & & & \\
\hline Coleodactylus brachystoma (Amaral, 1935) & $\mathrm{X}$ & $\mathrm{X}$ & CA, CE, PA & $\mathrm{X}^{*}$ & \\
\hline Coleodactylus cf. meridionalis (Boulenger, 1888) & & $\mathrm{X}$ & $\mathrm{CA}, \mathrm{CE}$ & $\mathrm{X}$ & \\
\hline Gonatodes cf. hasemani Griffin, 1917 & & $\mathrm{X}$ & FA, PA & $\mathrm{X}^{*}$ & $\mathrm{LC}$ \\
\hline Gonatodes humeralis (Guichenot, 1855) & $\mathrm{X}$ & $\mathrm{X}$ & $\mathrm{CE}, \mathrm{FA}, \mathrm{PA}$ & $\mathrm{X}^{*}$ & \\
\hline Mabuyidae (4) & & & & & \\
\hline Aspronema dorsivittatum (Cope, 1862) & $\mathrm{X}$ & $\mathrm{X}$ & $\mathrm{CE}, \mathrm{CH}, \mathrm{CS}, \mathrm{MA}, \mathrm{PA}$ & $\mathrm{X}$ & \\
\hline Copeoglossum nigropunctatum (Spix, 1825) & $\mathrm{X}$ & $\mathrm{X}$ & $\mathrm{CA}, \mathrm{CE}, \mathrm{CH}, \mathrm{FA}, \mathrm{MA}, \mathrm{PA}$ & $\mathrm{X}^{*}$ & \\
\hline Manciola guaporicola (Dunn, 1935) & $\mathrm{X}$ & $\mathrm{X}$ & $\mathrm{CE}, \mathrm{CH}, \mathrm{PA}$ & $\mathrm{X}^{*}$ & \\
\hline Notomabuya frenata (Cope, 1862) & $\mathrm{X}$ & $\mathrm{X}$ & $\mathrm{CA}, \mathrm{CE}, \mathrm{CH}, \mathrm{FA}, \mathrm{MA}, \mathrm{PA}$ & $X^{*}$ & \\
\hline Dactyloidae (2) & & & & & \\
\hline Norops aff. meridionalis & $\mathrm{X}$ & & PA & $\mathrm{X}^{*}$ & \\
\hline Norops meridionalis (Boettger, 1885) & $\mathrm{X}$ & $\mathrm{X}$ & $\mathrm{CE}, \mathrm{CH}, \mathrm{MA}, \mathrm{PA}$ & $\mathrm{X}^{*}$ & \\
\hline Hoplocercidae (1) & & & & & \\
\hline Hoplocercus spinosus Fitzinger, 1843 & & $\mathrm{X}$ & CA, CE, FA, PA & $\mathrm{X}^{*}$ & \\
\hline Iguanidae (1) & & & & & \\
\hline Iguana iguana (Linnaeus, 1758) & $\mathrm{X}$ & $\mathrm{X}$ & $\mathrm{CA}, \mathrm{CE}, \mathrm{CH}, \mathrm{FA}, \mathrm{MA}, \mathrm{PA}$ & $X^{*}$ & \\
\hline Polychrotidae (1) & & & & & \\
\hline Polychrus acutirostris Spix, 1825 & $\mathrm{X}$ & $\mathrm{X}$ & $\mathrm{CA}, \mathrm{CE}, \mathrm{CH}, \mathrm{MA}, \mathrm{PA}$ & $X^{*}$ & \\
\hline Tropiduridae (7) & & & & & \\
\hline Stenocercus caducus (Cope, 1862) & $\mathrm{X}$ & $\mathrm{X}$ & $\mathrm{CE}, \mathrm{CH}, \mathrm{MA}, \mathrm{PA}$ & $X^{*}$ & \\
\hline Tropidurus etheridgei Cei, 1982 & $\mathrm{X}$ & $\mathrm{X}$ & $\mathrm{CE}, \mathrm{CH}, \mathrm{PA}$ & $X^{*}$ & \\
\hline Tropidurus guarani Alvarez et al., 1994 & $\mathrm{X}$ & $\mathrm{X}$ & $\mathrm{CE}, \mathrm{CH}, \mathrm{MA}, \mathrm{PA}$ & $X^{*}$ & \\
\hline Tropidurus itambere Rodrigues, 1987 & $\mathrm{X}$ & $\mathrm{X}$ & CE, MA, PA & $\mathrm{X}$ & \\
\hline Tropidurus oreadicus Rodrigues, 1987 & & $\mathrm{X}$ & CE, MA, PA & $\mathrm{X}$ & \\
\hline Tropidurus spinulosus (Cope, 1862) & $\mathrm{X}$ & $\mathrm{X}$ & $\mathrm{CH}, \mathrm{PA}$ & $\mathrm{X}^{*}$ & \\
\hline Tropidurus torquatus (Wied, 1820) & $\mathrm{X}$ & $\mathrm{X}$ & $\mathrm{CE}, \mathrm{CH}, \mathrm{MA}$ & $X^{*}$ & $\mathrm{LC}$ \\
\hline Diploglossidae (2) & & & & & \\
\hline Ophiodes sp.1 - sensu Borges-Martins, 1998 & & $\mathrm{X}$ & CE, MA, PA & $\mathrm{X}$ & \\
\hline Ophiodes striatus (Spix, 1825) & $\mathrm{X}$ & $\mathrm{X}$ & $\mathrm{CE}, \mathrm{CH}, \mathrm{CS}, \mathrm{MA}, \mathrm{PA}$ & $\mathrm{X}^{*}$ & $\mathrm{LC}$ \\
\hline Gymnophthalmidae (9) & & & & & \\
\hline Bachia bresslaui (Amaral, 1935) & $\mathrm{X}$ & $\mathrm{X}$ & $\mathrm{CE}, \mathrm{CH}, \mathrm{MA}, \mathrm{PA}$ & $\mathrm{X}^{*}$ & VU \\
\hline
\end{tabular}


Tab. I. Cont.

\begin{tabular}{|c|c|c|c|c|c|}
\hline \multirow{2}{*}{ Táxon (número total de espécies) } & \multicolumn{2}{|c|}{ MR } & \multirow{2}{*}{ Domínios } & \multirow{2}{*}{$\mathrm{CZ}$} & \multirow{2}{*}{$\mathrm{EC}$} \\
\hline & PI & PL & & & \\
\hline Cercosaura ocellata Wagler, 1830 & & $\mathrm{X}$ & $\mathrm{CA}, \mathrm{CE}, \mathrm{CH}, \mathrm{CS}, \mathrm{FA}, \mathrm{MA}, \mathrm{PA}$ & $\mathrm{X}^{*}$ & \\
\hline Cercosaura parkeri Ruibal, 1952 & $\mathrm{X}$ & $\mathrm{X}$ & CE, PA & $\mathrm{X}^{*}$ & \\
\hline Cercosaura aff. schreibersii Wiegmann, 1834 & $\mathrm{X}$ & $\mathrm{X}$ & CE, PA & $X^{*}$ & \\
\hline Cercosaura cf. schreibersii Wiegmann, 1834 & $\mathrm{X}$ & $\mathrm{X}$ & $\mathrm{CE}, \mathrm{CH}, \mathrm{CS}, \mathrm{MA}, \mathrm{PA}$ & $X^{*}$ & $\mathrm{LC}$ \\
\hline Colobosaura modesta (Reinhardt \& Luetken, 1862) & $\mathrm{X}$ & $\mathrm{X}$ & $\mathrm{CA}, \mathrm{CE}, \mathrm{CH}, \mathrm{FA}, \mathrm{MA}, \mathrm{PA}$ & $\mathrm{X}^{*}$ & \\
\hline Micrablepharus atticolus Rodrigues, 1996 & $\mathrm{X}$ & $\mathrm{X}$ & $\mathrm{CE}, \mathrm{MA}, \mathrm{PA}$ & $\mathrm{X}^{*}$ & \\
\hline Micrablepharus maximiliani (Reinhardt \& Luetken, 1862) & $\mathrm{X}$ & $\mathrm{X}$ & $\mathrm{CA}, \mathrm{CE}, \mathrm{CH}, \mathrm{FA}, \mathrm{MA}, \mathrm{PA}$ & $X^{*}$ & \\
\hline Vanzosaura rubricauda (Boulenger, 1902) & $\mathrm{X}$ & $\mathrm{X}$ & $\mathrm{CA}, \mathrm{CE}, \mathrm{CH}, \mathrm{MA}, \mathrm{PA}$ & $X^{*}$ & \\
\hline \multicolumn{6}{|l|}{ Teiidae (14) } \\
\hline Ameiva sp. nov. & $\mathrm{X}$ & & PA & $\mathrm{X}^{*}$ & \\
\hline Ameiva ameiva (Linnaeus, 1758) & $\mathrm{X}$ & $\mathrm{X}$ & $\mathrm{CA}, \mathrm{CE}, \mathrm{CH}, \mathrm{FA}, \mathrm{MA}, \mathrm{PA}$ & $X^{*}$ & LC \\
\hline Ameivula aff. abalosi (Cabrera, 2012) & $\mathrm{X}$ & $\mathrm{X}$ & PA & $X^{*}$ & \\
\hline Ameivula jalapensis (Colli et al., 2009) & & $\mathrm{X}$ & $\mathrm{CA}, \mathrm{CE}$ & $X^{*}$ & \\
\hline Ameivula aff. ocellifera (Spix, 1825) & & $\mathrm{X}$ & CE, PA & $X^{*}$ & \\
\hline Dracaena paraguayensis Amaral, 1950 & $\mathrm{X}$ & $\mathrm{X}$ & $\mathrm{CE}, \mathrm{CH}, \mathrm{PA}$ & $X^{*}$ & \\
\hline Kentropyx paulensis Boettger, 1893 & & $\mathrm{X}$ & CE, FA, MA & $\mathrm{X}$ & \\
\hline Kentropyx vanzoi Gallagher \& Dixon, 1980 & & $\mathrm{X}$ & $\mathrm{CE}$ & $\mathrm{X}$ & \\
\hline Kentropyx viridistriga Boulenger, 1894 & $\mathrm{X}$ & $\mathrm{X}$ & $\mathrm{CE}, \mathrm{CH}, \mathrm{PA}$ & $\mathrm{X}^{*}$ & LC \\
\hline Kentropyx aff. viridistriga Boulenger, 1894 & $\mathrm{X}$ & $\mathrm{X}$ & $\mathrm{PA}$ & $\mathrm{X}^{*}$ & \\
\hline Salvator merianae (Duméril \& Bibron, 1839) & $\mathrm{X}$ & $\mathrm{X}$ & $\mathrm{CA}, \mathrm{CE}, \mathrm{CH}, \mathrm{CS}, \mathrm{MA}, \mathrm{PA}$ & $X^{*}$ & LC \\
\hline Teius teyou (Daudin, 1802) & $\mathrm{X}$ & $\mathrm{X}$ & $\mathrm{CE}, \mathrm{CH}, \mathrm{PA}$ & $X^{*}$ & \\
\hline Tupinambis quadrilineatus Manzani \& Abe, 1997 & & $\mathrm{X}$ & $\mathrm{CA}, \mathrm{CE}, \mathrm{MA}$ & & \\
\hline Tupinambis teguixin (Linnaeus, 1758) & $\mathrm{X}$ & $\mathrm{X}$ & CE, FA, PA & $X^{*}$ & \\
\hline Subtotais: & 37 & 46 & & $47\left(40^{*}\right)$ & \\
\hline \multicolumn{6}{|l|}{ SQUAMATA - AMPHISBAENIA (16) } \\
\hline \multicolumn{6}{|l|}{ Amphisbaenidae (16) } \\
\hline Amphisbaena alba Linnaeus, 1758 & $\mathrm{X}$ & $\mathrm{X}$ & $\mathrm{CA}, \mathrm{CE}, \mathrm{CH}, \mathrm{FA}, \mathrm{MA}, \mathrm{PA}$ & $\mathrm{X}^{*}$ & $\mathrm{LC}$ \\
\hline Amphisbaena anaemariae Vanzolini, 1997 & & $\mathrm{X}$ & $\mathrm{CE}$ & $\mathrm{X}$ & \\
\hline Amphisbaena bedai (Vanzolini, 1991) & $\mathrm{X}$ & $\mathrm{X}$ & CE, MA, PA & $\mathrm{X}^{*}$ & \\
\hline Amphisbaena camura Cope, 1862 & $\mathrm{X}$ & $\mathrm{X}$ & $\mathrm{CE}, \mathrm{CH}, \mathrm{PA}$ & $\mathrm{X}^{*}$ & \\
\hline Amphisbaena crisae Vanzolini, 1997 & & $\mathrm{X}$ & $\mathrm{CE}$ & $\mathrm{X}$ & \\
\hline Amphisbaena cuiabana (Strüssmann \& Carvalho, 2001) & $\mathrm{X}$ & & $\mathrm{CE}$ & $X^{*}$ & \\
\hline Amphisbaena leeseri Gans, 1964 & $\mathrm{X}$ & $\mathrm{X}$ & $\mathrm{CA}, \mathrm{CE}, \mathrm{CH}, \mathrm{MA}, \mathrm{PA}$ & $\mathrm{X}^{*}$ & \\
\hline Amphisbaena mertensii Strauch, 1881 & $\mathrm{X}$ & $\mathrm{X}$ & $\mathrm{CE}, \mathrm{CH}, \mathrm{MA}, \mathrm{PA}$ & $\mathrm{X}^{*}$ & \\
\hline Amphisbaena miringoera Vanzolini, 1971 & & $\mathrm{X}$ & CE, MA & & \\
\hline Amphisbaena pretrei Duméril \& Bibron, 1839 & & $\mathrm{X}$ & $\mathrm{CA}, \mathrm{CE}$ & & LC \\
\hline Amphisbaena roberti Gans, 1964 & & $\mathrm{X}$ & $\mathrm{CE}, \mathrm{CH}, \mathrm{MA}$ & $\mathrm{X}$ & \\
\hline Amphisbaena silvestrii Boulenger, 1902 & & $\mathrm{X}$ & $\mathrm{CE}$ & $X^{*}$ & \\
\hline Amphisbaena steindachneri Strauch, 1881 & $\mathrm{X}$ & & CE, MA & $\mathrm{X}^{*}$ & \\
\hline Amphisbaena vermicularis Wagler in Spix, 1824 & $\mathrm{X}$ & $\mathrm{X}$ & $\mathrm{CA}, \mathrm{CE}, \mathrm{MA}, \mathrm{PA}$ & $\mathrm{X}^{*}$ & \\
\hline Leposternon infraorbitale (Bertold, 1859) & & $\mathrm{X}$ & $\mathrm{CE}, \mathrm{MA}$ & & \\
\hline Leposternon microcephalum Wagler in Spix, 1824 & & $\mathrm{X}$ & $\mathrm{CA}, \mathrm{CE}, \mathrm{CH}, \mathrm{CS}, \mathrm{FA}, \mathrm{MA}, \mathrm{PA}$ & $X^{*}$ & \\
\hline Subtotais: & 8 & 14 & & $13\left(10^{*}\right)$ & \\
\hline \multicolumn{6}{|l|}{ SQUAMATA - SERPENTES (113) } \\
\hline \multicolumn{6}{|l|}{ Anomalepididae (3) } \\
\hline Liotyphlops beui (Amaral, 1924) & $\mathrm{X}$ & $\mathrm{X}$ & $\mathrm{CE}, \mathrm{CH}, \mathrm{MA}, \mathrm{PA}$ & $\mathrm{X}^{*}$ & $\mathrm{LC}$ \\
\hline Liotyphlops ternetzii (Boulenger, 1896) & & $\mathrm{X}$ & $\mathrm{CE}, \mathrm{CH}, \mathrm{FA}, \mathrm{MA}$ & $\mathrm{X}$ & \\
\hline Liotyphlops wilderi (Garman, 1883) & & $\mathrm{X}$ & CE, MA, PA & $\mathrm{X}$ & \\
\hline \multicolumn{6}{|l|}{ Typhlopidae (1) } \\
\hline Amerotyphlops brongersmianus (Vanzolini, 1976) & $\mathrm{X}$ & $\mathrm{X}$ & $\mathrm{CA}, \mathrm{CE}, \mathrm{CH}, \mathrm{CS}, \mathrm{FA}, \mathrm{MA}, \mathrm{PA}$ & $\mathrm{X}^{*}$ & \\
\hline \multicolumn{6}{|l|}{ Leptotyphlopidae (4) } \\
\hline Epictia clinorostris Arredondo \& Zaher, 2010 & & $\mathrm{X}$ & $\mathrm{CE}$ & $\mathrm{X}$ & \\
\hline Epictia vellardi (Laurent, 1984) & $\mathrm{X}$ & $\mathrm{X}$ & $\mathrm{CH}, \mathrm{PA}$ & $X^{*}$ & \\
\hline Trilepida brasiliensis (Laurent, 1949) & $\mathrm{X}$ & $\mathrm{X}$ & $\mathrm{CA}, \mathrm{CE}, \mathrm{PA}$ & $X^{*}$ & \\
\hline Trilepida koppesi (Amaral, 1955) & $\mathrm{X}$ & $\mathrm{X}$ & CE, PA & $\mathrm{X}^{*}$ & \\
\hline \multicolumn{6}{|l|}{ Boidae (5) } \\
\hline Boa constrictor Linnaeus, 1758 & $\mathrm{X}$ & $\mathrm{X}$ & $\mathrm{CA}, \mathrm{CE}, \mathrm{CH}, \mathrm{FA}, \mathrm{MA}, \mathrm{PA}$ & $X^{*}$ & \\
\hline Corallus hortulanus (Linnaeus, 1758) & $\mathrm{X}$ & & $\mathrm{CA}, \mathrm{CE}, \mathrm{FA}, \mathrm{MA}, \mathrm{PA}$ & $\mathrm{X}^{*}$ & \\
\hline Epicrates crassus Cope, 1862 & $\mathrm{X}$ & $\mathrm{X}$ & $\mathrm{CE}, \mathrm{CH}, \mathrm{MA}, \mathrm{PA}$ & $X^{*}$ & \\
\hline Eunectes murinus (Linnaeus, 1758) & $\mathrm{X}$ & $\mathrm{X}$ & $\mathrm{CA}, \mathrm{CE}, \mathrm{CH}, \mathrm{FA}, \mathrm{MA}, \mathrm{PA}$ & $\mathrm{X}^{*}$ & \\
\hline Eunectes notaeus Cope, 1862 & $\mathrm{X}$ & $\mathrm{X}$ & $\mathrm{CE}, \mathrm{CH}, \mathrm{PA}$ & $\mathrm{X}^{*}$ & \\
\hline Colubridae (15) & & & & & \\
\hline Chironius bicarinatus (Wied, 1820) & & $\mathrm{X}$ & $\mathrm{CA}, \mathrm{CE}, \mathrm{CH}, \mathrm{CS}, \mathrm{MA}, \mathrm{PA}$ & $X^{*}$ & \\
\hline Chironius exoletus (Linnaeus, 1758) & & $\mathrm{X}$ & $\mathrm{CE}, \mathrm{CH}, \mathrm{FA}, \mathrm{MA}, \mathrm{PA}$ & $X^{*}$ & \\
\hline Chironius flavolineatus (Jan, 1863) & $\mathrm{X}$ & $\mathrm{X}$ & $\mathrm{CA}, \mathrm{CE}, \mathrm{CH}, \mathrm{FA}, \mathrm{MA}, \mathrm{PA}$ & $X^{*}$ & \\
\hline
\end{tabular}


Tab. I. Cont.

\begin{tabular}{|c|c|c|c|c|c|}
\hline \multirow{2}{*}{ Táxon (número total de espécies) } & \multicolumn{2}{|c|}{ MR } & \multirow{2}{*}{ Domínios } & \multirow{2}{*}{$\mathrm{CZ}$} & \multirow{2}{*}{$\mathrm{EC}$} \\
\hline & PI & PL & & & \\
\hline Chironius fuscus (Linnaeus, 1758) & $\mathrm{X}$ & & FA, MA, PA & $\mathrm{X}^{*}$ & \\
\hline Chironius laurenti Dixon et al., 1993 & $\mathrm{X}$ & $\mathrm{X}$ & $\mathrm{CE}, \mathrm{PA}$ & $X^{*}$ & \\
\hline Chironius quadricarinatus (Boie, 1827) & $\mathrm{X}$ & $\mathrm{X}$ & $\mathrm{CA}, \mathrm{CE}, \mathrm{CH}, \mathrm{FA}, \mathrm{MA}, \mathrm{PA}$ & $X^{*}$ & \\
\hline Drymarchon corais (Boie, 1827) & $\mathrm{X}$ & $\mathrm{X}$ & $\mathrm{CA}, \mathrm{CE}, \mathrm{CH}, \mathrm{FA}, \mathrm{MA}, \mathrm{PA}$ & $X^{*}$ & \\
\hline Drymoluber brazili (Gomes, 1918) & $\mathrm{X}$ & $\mathrm{X}$ & $\mathrm{CA}, \mathrm{CE}, \mathrm{CH}, \mathrm{MA}, \mathrm{PA}$ & $X^{*}$ & \\
\hline Leptophis ahaetulla (Linnaeus, 1758) & $\mathrm{X}$ & $\mathrm{X}$ & $\mathrm{CA}, \mathrm{CE}, \mathrm{CH}, \mathrm{CS}, \mathrm{FA}, \mathrm{MA}, \mathrm{PA}$ & $\mathrm{X}^{*}$ & \\
\hline Mastigodryas bifossatus (Raddi, 1820) & $\mathrm{X}$ & $\mathrm{X}$ & $\mathrm{CA}, \mathrm{CE}, \mathrm{CH}, \mathrm{CS}, \mathrm{FA}, \mathrm{MA}, \mathrm{PA}$ & $X^{*}$ & \\
\hline Mastigodryas boddaerti (Sentzen, 1796) & $\mathrm{X}$ & $\mathrm{X}$ & CA, CE, FA, MA, PA & $X^{*}$ & \\
\hline Oxybelis aeneus (Wagler in Spix, 1824) & $\mathrm{X}$ & $\mathrm{X}$ & CA, CE, FA, MA, PA & $\mathrm{X}^{*}$ & \\
\hline Simophis rhinostoma (Schlegel, 1837) & $\mathrm{X}$ & $\mathrm{X}$ & $\mathrm{CE}, \mathrm{CH}, \mathrm{MA}, \mathrm{PA}$ & $X^{*}$ & \\
\hline Spilotes pullatus (Linnaeus, 1758) & $\mathrm{X}$ & $\mathrm{X}$ & $\mathrm{CA}, \mathrm{CE}, \mathrm{CH}, \mathrm{FA}, \mathrm{MA}, \mathrm{PA}$ & $X^{*}$ & \\
\hline Tantilla melanocephala (Linnaeus, 1758) & $\mathrm{X}$ & $\mathrm{X}$ & $\mathrm{CA}, \mathrm{CE}, \mathrm{CH}, \mathrm{CS}, \mathrm{FA}, \mathrm{MA}, \mathrm{PA}$ & $X^{*}$ & \\
\hline \multicolumn{6}{|l|}{ Dipsadidae $(75)^{* *}$} \\
\hline Apostolepis ambiniger (Peters, 1869) & $\mathrm{X}$ & & $\mathrm{CH}, \mathrm{PA}$ & $X^{*}$ & \\
\hline Apostolepis assimilis (Reinhardt, 1861) & & $\mathrm{X}$ & $\mathrm{CE}, \mathrm{CH}, \mathrm{MA}$ & $X^{*}$ & \\
\hline Apostolepis cf. christineae Lema, 2002 & $\mathrm{X}$ & & CE, PA & $X^{*}$ & \\
\hline Apostolepis dimidiata (Jan, 1862) & $\mathrm{X}$ & $\mathrm{X}$ & $\mathrm{CE}, \mathrm{CH}, \mathrm{MA}, \mathrm{PA}$ & $X^{*}$ & \\
\hline Apostolepis intermedia Koslowsky, 1898 & $\mathrm{X}$ & $\mathrm{X}$ & CE, PA & $X^{*}$ & \\
\hline Apostolepis goiasensis Prado 1942 & & $\mathrm{X}$ & $\mathrm{CE}$ & $\mathrm{X}$ & \\
\hline Atractus albuquerquei Cunha \& Nascimento, 1983 & & $\mathrm{X}$ & $\mathrm{CE}, \mathrm{FA}$ & $\mathrm{X}^{*}$ & $\mathrm{LC}$ \\
\hline Apostolepis aff. nigroterminata 1 Boulenger, 1896 & & $\mathrm{X}$ & PA & $\mathrm{X}^{*}$ & \\
\hline Apostolepis aff. nigroterminata 2 Boulenger, 1896 & & $\mathrm{X}$ & PA & $\mathrm{X}^{*}$ & \\
\hline Boiruna maculata (Boulenger, 1896) & $\mathrm{X}$ & & $\mathrm{CE}, \mathrm{CH}, \mathrm{CS}, \mathrm{MA}, \mathrm{PA}$ & $X^{*}$ & \\
\hline Clelia clelia (Daudin, 1803) & $\mathrm{X}$ & $\mathrm{X}$ & CH, FA, MA, PA & $X^{*}$ & \\
\hline Dipsas bucephala (Shaw, 1802) & $\mathrm{X}$ & & $\mathrm{CH}, \mathrm{PA}$ & $\mathrm{X}$ & \\
\hline Ditaxodon taeniatus (Peters in Hensel, 1868) & & $\mathrm{X}$ & CE, MA & $\mathrm{X}$ & \\
\hline Erythrolamprus aesculapii (Linnaeus, 1766) & $\mathrm{X}$ & $\mathrm{X}$ & $\mathrm{CA}, \mathrm{CE}, \mathrm{CH}, \mathrm{FA}, \mathrm{MA}, \mathrm{PA}$ & $X^{*}$ & $\mathrm{LC}$ \\
\hline Erythrolamprus almadensis (Wagler in Spix, 1824) & $\mathrm{X}$ & $\mathrm{X}$ & $\mathrm{CA}, \mathrm{CE}, \mathrm{CH}, \mathrm{CS}, \mathrm{FA}, \mathrm{MA}, \mathrm{PA}$ & $X^{*}$ & \\
\hline Erythrolamprus frenatus (Werner, 1909) & $\mathrm{X}$ & $\mathrm{X}$ & $\mathrm{CE}, \mathrm{CH}, \mathrm{MA}, \mathrm{PA}$ & $\mathrm{X}^{*}$ & \\
\hline Erythrolamprus jaegeri (Günther, 1858) & $\mathrm{X}$ & $\mathrm{X}$ & $\mathrm{CE}, \mathrm{CH}, \mathrm{CS}, \mathrm{MA}$ & $X^{*}$ & $\mathrm{LC}$ \\
\hline Erythrolamprus miliaris (Cope, 1868) & $\mathrm{X}$ & $\mathrm{X}$ & $\mathrm{CA}, \mathrm{CE}, \mathrm{CH}, \mathrm{CS}, \mathrm{FA}, \mathrm{MA}$ & $\mathrm{X}^{*}$ & \\
\hline Erythrolamprus poecilogyrus (Wied, 1825) & $\mathrm{X}$ & $\mathrm{X}$ & $\mathrm{CA}, \mathrm{CE}, \mathrm{CH}, \mathrm{CS}, \mathrm{FA}, \mathrm{MA}$ & $\mathrm{X}^{*}$ & \\
\hline Erythrolamprus reginae (Wagler, 1824) & $\mathrm{X}$ & $\mathrm{X}$ & $\mathrm{CA}, \mathrm{CE}, \mathrm{CH}, \mathrm{FA}, \mathrm{MA}, \mathrm{PA}$ & $X^{*}$ & \\
\hline Erythrolamprus taeniogaster (Jan, 1863) & & $\mathrm{X}$ & CA, CE, FA, MA & $\mathrm{X}^{*}$ & \\
\hline Erythrolamprus typhlus (Linnaeus, 1758) & $\mathrm{X}$ & $\mathrm{X}$ & $\mathrm{CA}, \mathrm{CE}, \mathrm{CH}, \mathrm{FA}, \mathrm{MA}, \mathrm{PA}$ & $\mathrm{X}^{*}$ & \\
\hline Helicops angulatus (Linnaeus, 1758) & $\mathrm{X}$ & $\mathrm{X}$ & CA, CE, FA, PA & $X^{*}$ & \\
\hline Helicops cf. carinicaudus (Wied, 1825) & & $\mathrm{X}$ & CE, MA & $\mathrm{X}^{*}$ & \\
\hline Helicops infrataeniatus (Jan, 1865) & & $\mathrm{X}$ & $\mathrm{CE}, \mathrm{CH}, \mathrm{CS}, \mathrm{MA}$ & $\mathrm{X}$ & \\
\hline Helicops leopardinus (Schlegel, 1837) & $\mathrm{X}$ & $\mathrm{X}$ & $\mathrm{CA}, \mathrm{CE}, \mathrm{CH}, \mathrm{CS}, \mathrm{FA}, \mathrm{PA}$ & $X^{*}$ & \\
\hline Helicops modestus Günther, 1861 & & $\mathrm{X}$ & CE, MA, PA & $X^{*}$ & \\
\hline Helicops polylepis Günther, 1861 & & $\mathrm{X}$ & CE, FA & $\mathrm{X}$ & \\
\hline Hydrodynastes bicinctus (Herrmann, 1804) & & $\mathrm{X}$ & CE, MA & $\mathrm{X}$ & \\
\hline Hydrodynastes gigas (Duméril et al.,1854) & $\mathrm{X}$ & $\mathrm{X}$ & $\mathrm{CA}, \mathrm{CE}, \mathrm{CH}, \mathrm{FA}, \mathrm{MA}, \mathrm{PA}$ & $\mathrm{X}^{*}$ & \\
\hline Hydrops caesurus Scrocchi et al., 2005 & $\mathrm{X}$ & $\mathrm{X}$ & $\mathrm{CE}, \mathrm{CH}, \mathrm{MA}, \mathrm{PA}$ & $\mathrm{X}^{*}$ & $\mathrm{LC}$ \\
\hline Imantodes cenchoa (Linnaeus, 1758) & & $\mathrm{X}$ & $\mathrm{CA}, \mathrm{CE}, \mathrm{CH}, \mathrm{FA}, \mathrm{MA}, \mathrm{PA}$ & $\mathrm{X}^{*}$ & \\
\hline Leptodeira annulata (Linnaeus, 1758) & $\mathrm{X}$ & $\mathrm{X}$ & $\mathrm{CA}, \mathrm{CE}, \mathrm{CH}, \mathrm{FA}, \mathrm{MA}, \mathrm{PA}$ & $X^{*}$ & \\
\hline Lygophis dilepis (Cope, 1862) & $\mathrm{X}$ & $\mathrm{X}$ & $\mathrm{CA}, \mathrm{CE}, \mathrm{CH}, \mathrm{MA}, \mathrm{PA}$ & $\mathrm{X}^{*}$ & $\mathrm{LC}$ \\
\hline Lygophis flavifrenatus (Cope, 1862) & & $\mathrm{X}$ & $\mathrm{CH}, \mathrm{CS}, \mathrm{MA}, \mathrm{PA}$ & $\mathrm{X}$ & \\
\hline Lygophis meridionalis (Schenkel, 1901) & $\mathrm{X}$ & $\mathrm{X}$ & $\mathrm{CA}, \mathrm{CE}, \mathrm{CH}, \mathrm{MA}, \mathrm{PA}$ & $X^{*}$ & \\
\hline Mussurana bicolor (Peracca, 1904) & $\mathrm{X}$ & $\mathrm{X}$ & CE, CH, MA, PA & $\mathrm{X}^{*}$ & $\mathrm{LC}$ \\
\hline Oxyrhopus guibei Hoge \& Romano, 1978 & & $\mathrm{X}$ & $\mathrm{CA}, \mathrm{CE}, \mathrm{CH}, \mathrm{MA}, \mathrm{PA}$ & $X^{*}$ & \\
\hline Oxyrhopus petolarius (Linnaeus, 1758) & $\mathrm{X}$ & $\mathrm{X}$ & $\mathrm{CE}, \mathrm{CH}, \mathrm{FA}, \mathrm{MA}, \mathrm{PA}$ & $X^{*}$ & \\
\hline Oxyrhopus rhombifer Duméril et al.,1854 & $\mathrm{X}$ & $\mathrm{X}$ & $\mathrm{CA}, \mathrm{CE}, \mathrm{CH}, \mathrm{CS}, \mathrm{FA}, \mathrm{MA}, \mathrm{PA}$ & $\mathrm{X}^{*}$ & \\
\hline Oxyrhopus trigeminus Duméril et al., 1854 & $\mathrm{X}$ & $\mathrm{X}$ & CA, CE, FA, MA, PA & $X^{*}$ & \\
\hline Paraphimophis rustica (Cope, 1878) & & $\mathrm{X}$ & $\mathrm{CE}, \mathrm{CH}, \mathrm{CS}, \mathrm{MA}, \mathrm{PA}$ & $\mathrm{X}$ & \\
\hline Phalotris matogrossensis Lema et al., 2005 & $\mathrm{X}$ & $\mathrm{X}$ & $\mathrm{CE}, \mathrm{CH}, \mathrm{MA}, \mathrm{PA}$ & $\mathrm{X}^{*}$ & $\mathrm{LC}$ \\
\hline Phalotris mertensi (Hoge, 1955) & & $\mathrm{X}$ & CE, MA, PA & $X^{*}$ & \\
\hline Phalotris multipunctatus Puorto \& Ferrarezzi, 1994 & & $\mathrm{X}$ & $\mathrm{CE}, \mathrm{MA}$ & $\mathrm{X}$ & \\
\hline Phalotris nasutus (Gomes, 1915) & $\mathrm{X}$ & $\mathrm{X}$ & CE, FA, MA, PA & $X^{*}$ & \\
\hline Phalotris tricolor (Duméril et al., 1854) & & $\mathrm{X}$ & $\mathrm{CE}, \mathrm{CH}, \mathrm{MA}, \mathrm{PA}$ & $X^{*}$ & $\mathrm{LC}$ \\
\hline Philodryas livida (Amaral, 1923) & $\mathrm{X}$ & $\mathrm{X}$ & CE, MA, PA & $\mathrm{X}$ & VU \\
\hline Philodryas mattogrossensis Koslowsky, 1898 & $\mathrm{X}$ & $\mathrm{X}$ & $\mathrm{CE}, \mathrm{CH}, \mathrm{MA}, \mathrm{PA}$ & $\mathrm{X}^{*}$ & \\
\hline Philodryas nattereri Steindachner, 1870 & $\mathrm{X}$ & $\mathrm{X}$ & CA, CE, MA, PA & $X^{*}$ & \\
\hline Philodryas olfersii (Lichtenstein, 1823) & $\mathrm{X}$ & $\mathrm{X}$ & $\mathrm{CA}, \mathrm{CE}, \mathrm{CH}, \mathrm{CS}, \mathrm{FA}, \mathrm{MA}, \mathrm{PA}$ & $X^{*}$ & \\
\hline Philodryas patagoniensis (Girard, 1858) & $\mathrm{X}$ & $\mathrm{X}$ & CA, CE, CH, CS, MA, PA & $X^{*}$ & \\
\hline
\end{tabular}


Tab. I. Cont.

\begin{tabular}{|c|c|c|c|c|c|}
\hline \multirow{2}{*}{ Táxon (número total de espécies) } & \multicolumn{2}{|c|}{ MR } & \multirow{2}{*}{ Domínios } & \multirow{2}{*}{$\mathrm{CZ}$} & \multirow{2}{*}{$\mathrm{EC}$} \\
\hline & PI & PL & & & \\
\hline Philodryas psammophidea Günther, 1872 & & $\mathrm{X}$ & $\mathrm{CE}, \mathrm{CH}, \mathrm{MA}, \mathrm{PA}$ & $\mathrm{X}$ & LC \\
\hline Phimophis guerini (Duméril et al., 1854) & $\mathrm{X}$ & $\mathrm{X}$ & $\mathrm{CA}, \mathrm{CE}, \mathrm{CH}, \mathrm{MA}, \mathrm{PA}$ & $\mathrm{X}^{*}$ & \\
\hline Pseudoboa coronata Schneider, 1801 & $\mathrm{X}$ & $\mathrm{X}$ & CE, FA, PA & $X^{*}$ & \\
\hline Pseudoboa neuwiedii (Duméril et al., 1854) & $\mathrm{X}$ & $\mathrm{X}$ & CE, FA, MA, PA & $\mathrm{X}$ & \\
\hline Pseudoboa nigra (Duméril et al., 1854) & $\mathrm{X}$ & $\mathrm{X}$ & $\mathrm{CA}, \mathrm{CE}, \mathrm{CH}, \mathrm{FA}, \mathrm{MA}, \mathrm{PA}$ & $X^{*}$ & \\
\hline Pseudoeryx plicatilis (Linnaeus, 1758) & $\mathrm{X}$ & $\mathrm{X}$ & $\mathrm{CE}, \mathrm{CH}, \mathrm{FA}, \mathrm{MA}, \mathrm{PA}$ & $X^{*}$ & $\mathrm{LC}$ \\
\hline Psomophis genimaculatus (Boettger, 1885) & $\mathrm{X}$ & $\mathrm{X}$ & $\mathrm{CE}, \mathrm{CH}, \mathrm{MA}, \mathrm{PA}$ & $X^{*}$ & \\
\hline Rhachidelus brazili Boulenger, 1908 & & $\mathrm{X}$ & $\mathrm{CE}, \mathrm{CH}, \mathrm{MA}, \mathrm{PA}$ & $\mathrm{X}$ & \\
\hline Sibynomorphus lavillai Scrocchi et al., 1993 & & $\mathrm{X}$ & $\mathrm{CE}, \mathrm{CH}, \mathrm{PA}$ & $X^{*}$ & \\
\hline Sibynomorphus mikanii (Schlegel, 1837) & $\mathrm{X}$ & $\mathrm{X}$ & $\mathrm{CA}, \mathrm{CE}, \mathrm{MA}, \mathrm{PA}$ & $X^{*}$ & \\
\hline Sibynomorphus turgidus (Cope, 1868) & $\mathrm{X}$ & $\mathrm{X}$ & $\mathrm{CE}, \mathrm{CH}, \mathrm{CS}, \mathrm{FA}, \mathrm{MA}, \mathrm{PA}$ & $X^{*}$ & \\
\hline Sibynomorphus ventrimaculatus (Boulenger, 1885) & $\mathrm{X}$ & $\mathrm{X}$ & $\mathrm{CE}, \mathrm{CH}, \mathrm{CS}, \mathrm{MA}, \mathrm{PA}$ & $X^{*}$ & $\mathrm{LC}$ \\
\hline Taeniophallus occipitalis (Jan, 1863) & $\mathrm{X}$ & $\mathrm{X}$ & $\mathrm{CA}, \mathrm{CE}, \mathrm{CH}, \mathrm{CS}, \mathrm{FA}, \mathrm{MA}, \mathrm{PA}$ & $\mathrm{X}^{*}$ & \\
\hline Thamnodynastes chaquensis Bergna \& Alvarez, 1993 & $\mathrm{X}$ & $\mathrm{X}$ & $\mathrm{CE}, \mathrm{CH}, \mathrm{PA}$ & $X^{*}$ & \\
\hline Thamnodynastes hypoconia (Cope, 1860) & $\mathrm{X}$ & $\mathrm{X}$ & $\mathrm{CA}, \mathrm{CE}, \mathrm{CH}, \mathrm{CS}, \mathrm{FA}, \mathrm{MA}, \mathrm{PA}$ & $X^{*}$ & \\
\hline Thamnodynastes lanei Bailey et al., 2005 & $\mathrm{X}$ & $\mathrm{X}$ & $\mathrm{CE}, \mathrm{CH}, \mathrm{PA}$ & $X^{*}$ & \\
\hline Thamnodynastes rutilus (Prado, 1942) & & $\mathrm{X}$ & $\mathrm{CE}, \mathrm{MA}$ & $\mathrm{X}$ & \\
\hline Tomodon dorsatus Duméril et al., 1854 & $\mathrm{X}$ & & $\mathrm{CE}, \mathrm{CH}, \mathrm{CS}, \mathrm{MA}, \mathrm{PA}$ & & \\
\hline Xenodon matogrossensis (Scrocchi \& Cruz, 1993) & $\mathrm{X}$ & $\mathrm{X}$ & $\mathrm{CE}, \mathrm{CH}, \mathrm{PA}$ & $\mathrm{X}^{*}$ & \\
\hline Xenodon merremii (Wagler in Spix, 1824) & $\mathrm{X}$ & $\mathrm{X}$ & $\mathrm{CA}, \mathrm{CE}, \mathrm{CH}, \mathrm{CS}, \mathrm{FA}, \mathrm{MA}, \mathrm{PA}$ & $X^{*}$ & \\
\hline Xenodon nattereri (Steindachner, 1867) & & $\mathrm{X}$ & $\mathrm{CE}, \mathrm{MA}$ & $\mathrm{X}$ & \\
\hline Xenodon rhabdocephalus (Wied, 1824) & & $\mathrm{X}$ & CE, FA, MA & $\mathrm{X}$ & \\
\hline Xenopholis werdingorum Jansen et al., 2009 & & $\mathrm{X}$ & $\mathrm{CH}, \mathrm{PA}$ & $X^{*}$ & \\
\hline \multicolumn{6}{|l|}{ Elapidae (5) } \\
\hline Micrurus corallinus (Merrem, 1820) & & $\mathrm{X}$ & $\mathrm{CE}, \mathrm{CH}, \mathrm{MA}$ & & \\
\hline Micrurus frontalis (Duméril et al., 1854) & $\mathrm{X}$ & $\mathrm{X}$ & $\mathrm{CE}, \mathrm{CH}, \mathrm{MA}, \mathrm{PA}$ & $X^{*}$ & \\
\hline Micrurus lemniscatus (Linnaeus, 1758) & & $\mathrm{X}$ & $\mathrm{CA}, \mathrm{CE}, \mathrm{FA}, \mathrm{MA}$ & $\mathrm{X}^{*}$ & \\
\hline Micrurus pyrrhocryptus (Cope, 1862) & $\mathrm{X}$ & $\mathrm{X}$ & $\mathrm{CH}, \mathrm{PA}$ & $X^{*}$ & $\mathrm{LC}$ \\
\hline Micrurus tricolor Hoge, 1956 & $\mathrm{X}$ & $\mathrm{X}$ & $\mathrm{CE}, \mathrm{CH}, \mathrm{MA}, \mathrm{PA}$ & $\mathrm{X}$ & \\
\hline \multicolumn{6}{|l|}{ Viperidae (5) } \\
\hline Bothrops alternatus Duméril et al., 1854 & & $\mathrm{X}$ & $\mathrm{CA}, \mathrm{CE}, \mathrm{CH}, \mathrm{CS}, \mathrm{MA}$ & $\mathrm{X}^{*}$ & \\
\hline Bothrops mattogrossensis Amaral, 1925 & $\mathrm{X}$ & $\mathrm{X}$ & $\mathrm{CA}, \mathrm{CE}, \mathrm{CH}, \mathrm{MA}, \mathrm{PA}$ & $\mathrm{X}^{*}$ & \\
\hline Bothrops moojeni Hoge, 1966 & $\mathrm{X}$ & $\mathrm{X}$ & $\mathrm{CA}, \mathrm{CE}, \mathrm{CH}, \mathrm{MA}, \mathrm{PA}$ & $\mathrm{X}^{*}$ & \\
\hline Bothrops pauloensis Amaral, 1925 & $\mathrm{X}$ & $\mathrm{X}$ & $\mathrm{CA}, \mathrm{CE}, \mathrm{CH}, \mathrm{MA}, \mathrm{PA}$ & $\mathrm{X}^{*}$ & \\
\hline Crotalus durissus (Laurenti, 1768) & $\mathrm{X}$ & $\mathrm{X}$ & $\mathrm{CA}, \mathrm{CE}, \mathrm{CH}, \mathrm{CS}, \mathrm{FA}, \mathrm{MA}, \mathrm{PA}$ & $X^{*}$ & $\mathrm{LC}$ \\
\hline Subtotais: & 79 & 106 & & $111(91 *)$ & \\
\hline Total: 188 espécies & 131 & 176 & & $178(147 *)$ & \\
\hline
\end{tabular}

coleções científicas não regionais. Contudo, e apesar do crescente número de novas ocorrências para o MS (e.g., ArRudA et al., 2011; LANDGREF Filho \& AOKI, 2011; BARreto et al., 2012; SugAi et al., 2012), a riqueza de répteis do estado ainda está, sem dúvida, subestimada. Cerca de $25 \%$ das espécies de répteis do Brasil (segundo CosTA \& BÉRNILS, 2014) tiveram seus registros confirmados para o estado. Considerando que o MS representa menos de $4 \%$ do território nacional, a riqueza do estado, mesmo que subestimada, é notável, e pode estar relacionada à diversidade de ambientes e ao intercâmbio da fauna de domínios vizinhos (AdÁmoli, 1982; Prance \& Schaller, 1982; Alho et al., 2001). A riqueza de répteis do MS também é expressiva quando comparada a outros estados que, em consequência da ocupação humana, possuem estudos mais frequentes e antigos. Por exemplo, São Paulo (SP) e Rio de Janeiro (RJ), mesmo sendo menores $(2,9 \%$ e $0,5 \%$ do território nacional, respectivamente) abrigam riquezas que se aproximam da apresentada atualmente para o MS ( $\mathrm{SP}=28 \%$; RJ $=17 \%$ ) (RochA et al., 2004; ZAHER et al., 2012). Esse cenário indica o potencial de aumento da riqueza conhecida de répteis no MS à medida que os estudos no estado forem intensificados.
A contribuição relativa de cada ordem para a riqueza de espécies do MS é muito semelhante à registrada para o Brasil (COSTA \& BÉRNILs, 2014), com Squamata perfazendo a maioria das espécies (94\%), sendo serpentes o grupo mais rico, seguido de Sauria e Amphisbaenia. Três espécies de Crocodylia, das seis encontradas no Brasil, ocorrem no MS, provavelmente em consequência do intercâmbio de espécies com regiões vizinhas (ALHO et al., 2001), heterogeneidade e abundância de ambientes aquáticos.

A riqueza e composição de espécies variaram entre macrorregiões, tendo sido registradas 176 espécies no planalto, das quais $30,7 \%$ foram unicamente registradas neste; e 131 na planície de inundação, onde $6 \%$ foram exclusivas. Dentre os domínios, o Cerrado é o mais rico em espécies ( $n=171)$, seguido pelo Pantanal $(n=151)$, Mata Atlântica $(n=133)$ e Chaco $(n=119)$, sendo que muitas espécies são comuns a mais de um domínio.

O padrão de riqueza de répteis encontrado, com a planície apresentando menor número de espécies do que as áreas de planalto, já foi registrado para os répteis da BAP, e para diversos outros grupos animais (STRÜSSMANN et al., 2000; JuNK et al., 2006). Esse fato geralmente é atribuído 
às origens geológicas recentes do Pantanal e aos ciclos de cheia e seca, que impõem inúmeras restrições para a colonização e estabelecimento de espécies vindas de áreas vizinhas (AB'SABER, 1988; AlHO et al., 2001). Em contraste, a macrorregião de planalto do MS abrange, originalmente, porções dos domínios da Mata Atlântica, com fitofisionomias particulares e espécies a elas associadas, além do Cerrado com sua elevada taxa de endemismo e riqueza de espécies (NoGUEIRA et al., 2011), o que pode explicar a elevada riqueza encontrada no Cerrado do MS, mesmo sendo o local com maiores lacunas de amostragem.

Com exceção da sua porção centro-oeste, a fauna de répteis da maior parte do MS é muito pouco conhecida (Fig. 1). No norte, sul e leste do estado não há nenhuma área bem estudada, incluindo UCs e RPPNs (Fig. 2).

Muitas áreas do estado ainda não foram adequadamente amostradas quanto à fauna de répteis. Apesar de a porção centro-oeste ser a mais conhecida, e isso inclui a planície de inundação, a dificuldade de acesso a certas regiões (e.g., áreas quase permanentemente alagadas como a sub-região conhecida como Pantanal do Paiaguás, Ротт \& AdÁmoli, 1999), associada à carência de recursos humanos, parecem ser alguns dos maiores obstáculos para o desenvolvimento de estudos. Já a sub-região do Pantanal do Miranda (sensu
HAMILTON et al., 1996) é apontada como a mais bem estudada do MS (StrüsSMAnN et al., 2007). Essa concentração de amostragens é atribuída à atuação de pesquisadores locais e facilidade de acesso. Todavia, nos últimos anos houve um progresso considerável na distribuição dos estudos e, no presente trabalho, parte da sub-região do Pantanal da Nhecolândia (sensu Hamilton et al., 1996) também foi considerada como uma das mais bem amostradas. Da mesma forma, houve um incremento do conhecimento em áreas do planalto, em decorrência dos estudos para elaboração de planos de manejo e da divulgação de informações sobre a fauna em unidades de conservação como RPPNs e Parque Nacional da Serra de Bodoquena (ver revisão em HARRIS et al., 2005b; UetanABARo et al., 2007).

Cerca de $40 \%$ das espécies de répteis do MS tem ampla distribuição, ocorrendo em pelo menos cinco dos sete domínios do país. Somente $22 \%$ dos répteis são restritos a um ou dois domínios (Tab. I). A composição de répteis do estado é mais parecida com a fauna chaquenha (63\%), seguido pelo Cerrado e Mata Atlântica, ambos com aproximadamente 50\% de semelhança. A similaridade com os domínios presentes na diagonal de formações abertas da América do Sul é de $40 \%$, sendo a Caatinga a mais distinta dentre os três domínios (Fig. 3).

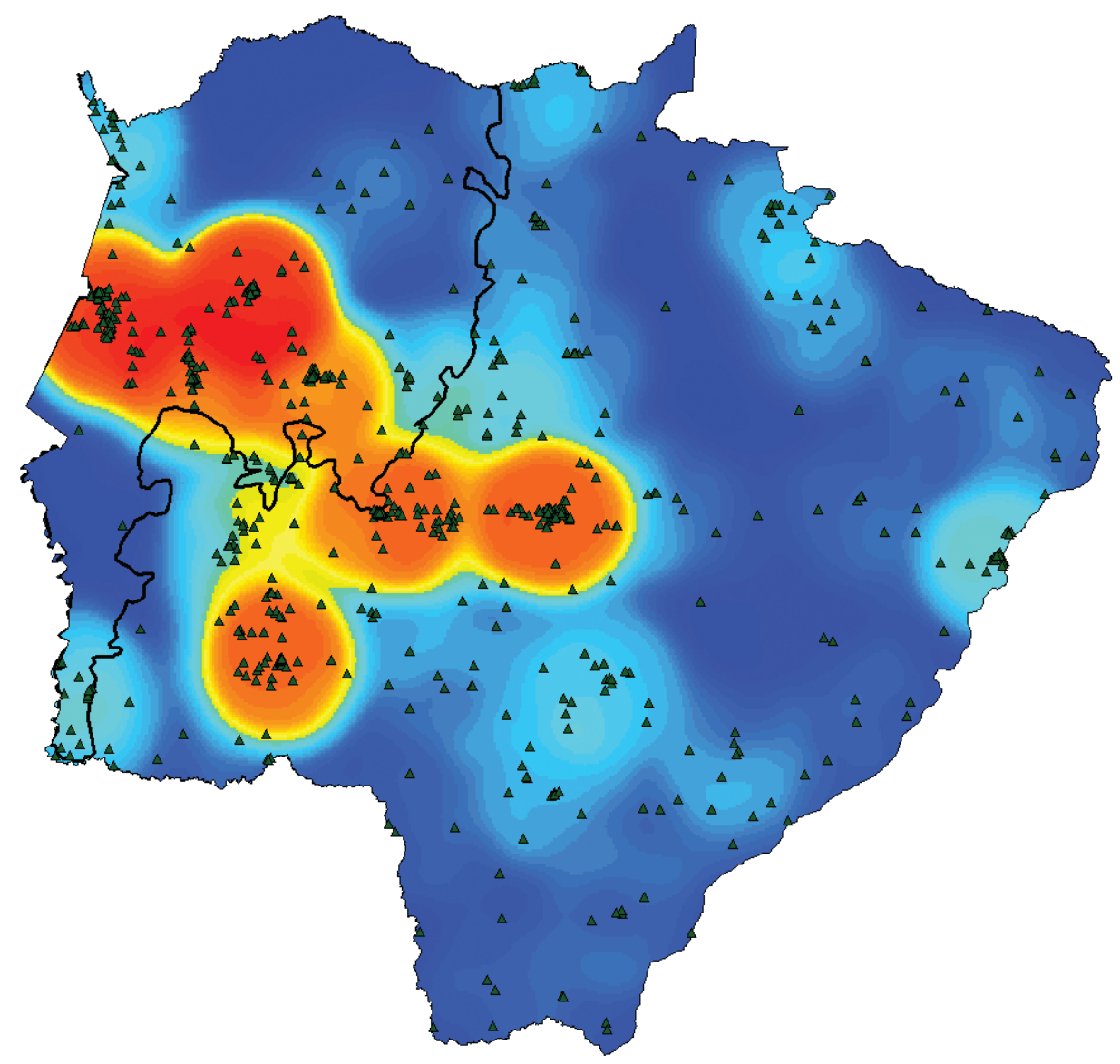

Fig. 1. Distribuição das amostragens de répteis no estado de Mato Grosso do Sul, Brasil (triângulos escuros) e zonas de conhecimento ou amostragens de répteis geradas pela interpolação circular neighbourhood no programa Diva-Gis. As cores indicam um gradiente do grau de conhecimento que varia do vermelho (grau máximo) ao azul escuro (mínimo). Linha negra: separação entre as macrorregiões tratadas neste estudo (a oeste - Planície de inundação; leste - Planalto). 


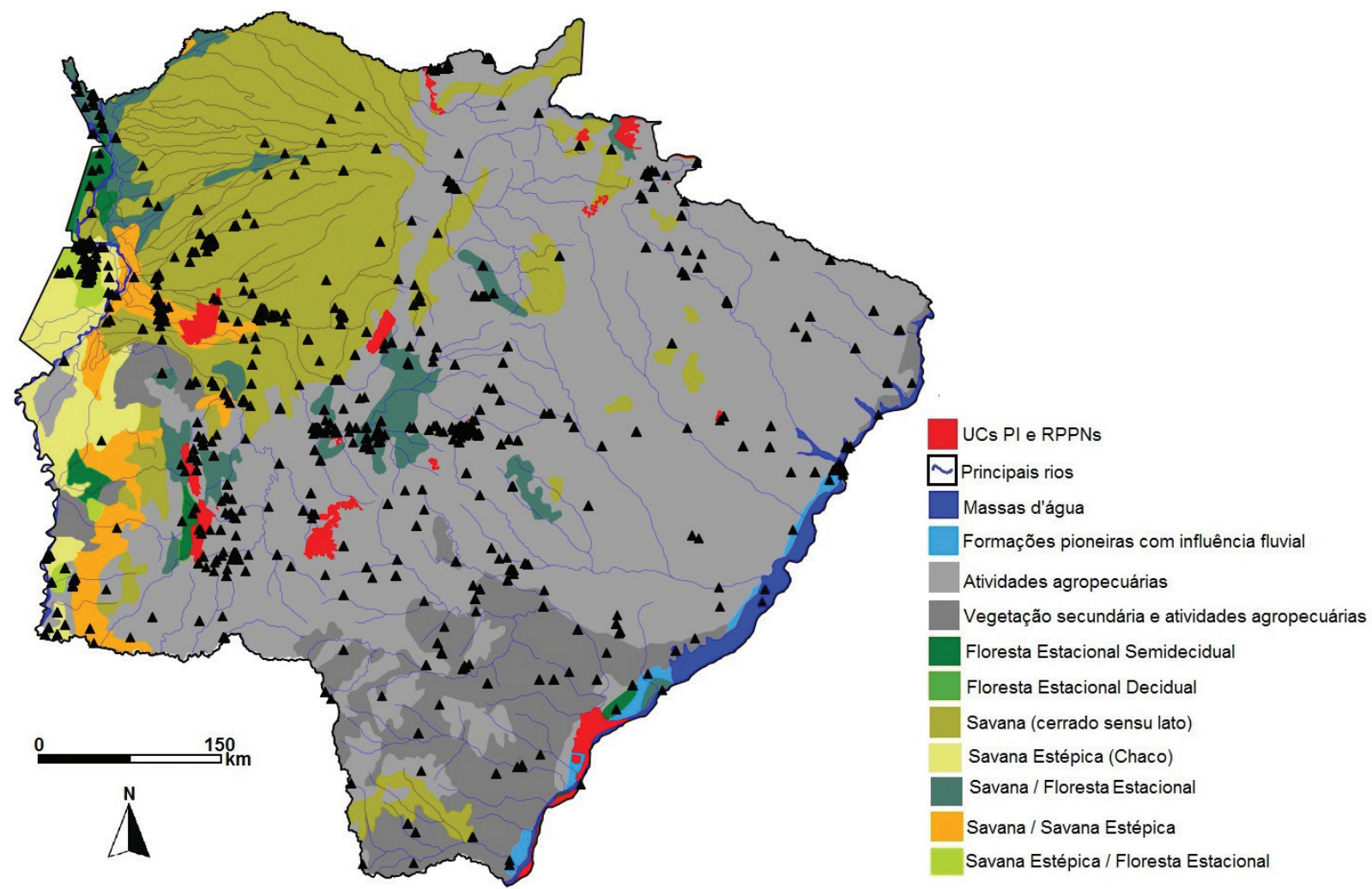

Fig. 2. Distribuição das amostragens de répteis no estado de Mato Grosso do Sul, Brasil (triângulos escuros) nos tipos vegetacionais e nas unidades de conservação (UCsPI) e reservas particulares do patrimônio natural (RPPNs) de acordo com IBGE (para detalhes, ver material e métodos).

O MS compartilha espécies de répteis com todos os domínios brasileiros, e quase a metade dos répteis do estado tem ampla distribuição nesses domínios. O local mais bem amostrado e cerca de um quarto do estado estão sob o domínio do Pantanal, local de formação geológica recente (Assine, 2003) e sem registro de endemismo de espécies (StRÜSSMANN et al., 2007). Entretanto, esse cenário pode mudar, pois o número de estudos taxonômicos no estado vem aumentando, e já se sabe que algumas espécies são de ocorrência restrita a determinado domínio pelo menos no MS. Ainda, com o incremento de estudos nas regiões apontadas como lacunas de amostragem, em especial em locais de Mata Atlântica e Cerrado, pode ser que essa proporção não se mantenha uma vez que espécies típicas desses domínios podem vir a ser registradas.

No estado, apesar do Chaco ocupar pequena extensão territorial e o único estudo sobre sua herpetofauna ser pontual (SouZa et al., 2010), o conjunto de espécies desse domínio é muito semelhante ao encontrado no Pantanal. Como a região mais bem inventariada no MS está no Pantanal, é possível sugerir que a elevada similaridade do MS com o Chaco é resultado da maior proximidade da fauna de répteis deste com o Pantanal. Ainda que o Cerrado seja o domínio fitogeográfico predominante no estado, sua similaridade com o MS é inferior ao Chaco. O Cerrado apresenta extensão muito ampla no Brasil e é, em geral, relativamente bem amostrado, entretanto, no MS as maiores lacunas de conhecimento estão localizadas nesse domínio. Dessa forma, a porção

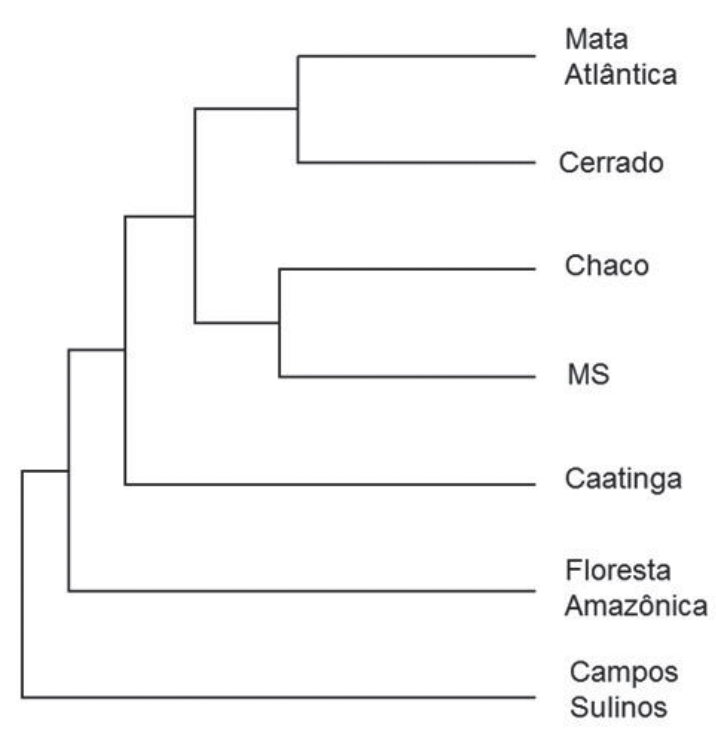

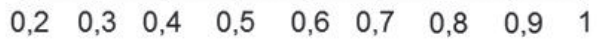

Fig. 3. Análise de classificação pelo método de agrupamento UPGMA, utilizando o índice de dissimilaridade de Sorensen, entre a fauna de répteis do Mato Grosso do Sul, Brasil (presença e ausência de espécies) e a encontrada nos domínios fitogeográficos do Brasil e no domínio do Chaco.

de Cerrado no MS não tem sua real riqueza conhecida, compartilhando assim menor número de espécies com esse domínio. De modo geral, a fauna estadual de répteis é mais parecida com aquela encontrada ao longo de toda a diagonal 
de formações abertas. Dentre os domínios que formam essa diagonal, o MS compartilha menos espécies com a Caatinga, o que pode ser justificado pela distância, ausência de áreas de contato e fatores históricos. O padrão de semelhança ecológica (e não histórico) entre os domínios do Cerrado, Chaco e Caatinga pode também ser verificado em outros grupos animais (ZANELLA, 2002; COLLI, 2005; PoRZECANSKI \& CRACRAFT, 2005). A estreita relação entre a fauna desses domínios necessita ser explorada para esclarecer se as relações refletem maior número de espécies compartilhadas ou uma história compartilhada (WERNECK, 2011). Ainda, é preciso lembrar que o estado apresenta mais lacunas de conhecimento que áreas minimamente conhecidas.

Síntese zoogeográfica e taxonômica. Espécies recentemente descritas (últimos dez anos). Sauria: Ameivula jalapensis; Serpentes: Epictia clinorostris, Hydrops caesurus, Phalotris matogrossensis, Thamnodynastes lanei, Xenopholis werdingorum.

Espécies novas para a ciência, em processo de descrição formal (sp. nov. ou aff.). Sauria: Ameiva sp. nov., Ameivula aff. abalosi, Ameivula aff. ocellifera, Cercosaura aff. schreibersii, Kentropyx aff. viridistriga, Norops aff. meridionalis, Ophiodes sp.1.; Serpentes: Apostolepis aff. nigroterminata 1, Apostolepis aff. nigroterminata 2.

Ampliações de distribuição (últimos dez anos). Sauria: Ameivula jalapensis, Bachia bresslaui, Cercosaura parkeri, Tupinambis quadrilineatus; Amphisbaenia: Amphisbaena cuiabana, A. steindachneri; Serpentes: Apostolepis goiasensis, Epictia vellardi, Phalotris nasutus, Rhachidelus brazili, Sibynomorphus lavillai.

Espécies que, dentro do estado, possuem distribuição restrita (extensão de ocorrência conhecida no MS $<20.000 \mathrm{~km}^{2}$ no Brasil). Sauria: Ameiva sp. nov., Ameivula aff. abalosi, Teius teyou, Tropidurus spinulosus; Amphisbaenia: Amphisbaena cuiabana, A. steindachneri; Serpentes: Apostolepis aff. nigroterminata 1, Apostolepis aff. nigroterminata 2, Apostolepis intermedia, Phalotris multipunctatus, Phalotris tricolor, Sibynomorphus lavillai.

Espécies com localidade-tipo no MS. Sauria: Lygodactylus wetzeli (Corumbá), Ophiodes sp. 1 (sensu Borges-Martins) (Anastácio); Amphisbaenia: Amphisbaena bedai (Anastácio), A. leeseri (Corumbá); Serpentes: Apostolepis intermedia (Miranda), Bothrops mattogrossensis (Miranda), Micrurus tricolor (Carandazal), Phalotris multipunctatus (Rio Brilhante), Philodryas mattogrossensis (Miranda), Thamnodynastes lanei (Salobra), Trilepida koppesi (Terenos), Xenodon matogrossensis (MS).

Espécies de ocorrência até o momento restrita, no Brasil, ao MS. Sauria: Ameiva sp.nov., Ameivula aff. abalosi, Teius teyou, Tropidurus spinulosus; Amphisbaenia: Amphisbaena bedai, A. camura, A. leeseri; Serpentes: Phalotris tricolor, Apostolepis aff. nigroterminata 1, Apostolepis aff. nigroterminata 2, Apostolepis intermedia e Sibynomorphus lavillai.

Espécies exóticas para o MS. Sauria: Hemidactylus mabouia (África); Testudines: Podocnemis unifilis (Floresta
Amazônica e Cerrado), Trachemys scripta (América do Norte).

Espécies não incluídas na lista por falta de confirmação de material testemunho. Sauria: Norops chrysolepis (em Souza et al., 2010), Tropidurus hispidus, Varzea bistriata (em Silva JR. et al., 2009); Serpentes: Bothrops neuwiedi (em Silva JR. et al., 2009), Gomesophis brasiliensis (em SILVA JR. et al., 2009), Siagonodon septemstriatus (em SILVA JR. et al., 2009), Sordelina punctata (PEREIRA et al., 2007), Thamnodynastes pallidus e T. strigatus (em SiLVA JR. et al., 2009).

Espécie de ocorrência duvidosa: Ditaxodon taeniatus (ver THOMAs et al., 2006).

Espécies consideradas vulneráveis (VU) pela IUCN (2012). Testudines: Acanthochelys macrocephala, Chelonoidis denticulata, Mesoclemmys vanderhaegei, Podocnemis unifilis; Sauria: Bachia bresslaui; Serpentes: Philodryas livida. Esta última também foi incluída como vulnerável na Lista das Espécies da fauna Brasileira Ameaçadas de Extinção, recentemente atualizada (ICMBio, 2015). Dentre os répteis registrados para o MS figuram nesta lista, ainda, as serpentes Ditaxodon taeniatus (VU) e Phalotris multipunctatus (Em perigo - EN), e o lagarto Kentropyx vanzoi (VU).

Principais grupos de pesquisa no estado do Mato Grosso do Sul. O MS conta com somente dois Programas de Pós-graduação com linhas de pesquisa que permitem a inserção de estudos com répteis, ambos vinculados à Universidade Federal do Mato Grosso do Sul: PPG Ecologia e Conservação (mestrado e doutorado) - linhas de pesquisa: ecologia aplicada, da reprodução, das adaptações, de comunidades, de ecossistemas e de populações; PPG Biologia Animal (mestrado) - linhas de pesquisa: morfofisiologia e fisiopatologia animal, sistemática e bionomia animal.

O mapa de investimentos do CNPq indica que 11,8\% dos recursos nacionais atualmente destinados à pesquisa estão alocados no MS, dentre os quais 7,2\% destinam-se a projetos individuais e de desenvolvimento científico regional.

Foram identificados oito grupos de pesquisa que envolvem estudos sobre répteis do MS, certificados por quatro instituições de ensino superior federal e privado (Universidade Católica Dom Bosco, UCDB; Universidade Federal da Grande Dourados, UFGD; Universidade Federal do Mato Grosso do Sul, UFMS e Universidade para o Desenvolvimento do Estado e da Região do Pantanal, Anhanguera, UNIDERP). Entretanto, em apenas dois desses grupos de pesquisa, ambos vinculados à UFMS, os répteis estão entre os principais alvos de investigação ("História Natural de Anfíbios e Répteis" e "História Natural e Ecologia de Vertebrados em Ambientes Naturais e Antrópicos").

Principais acervos no estado do Mato Grosso do Sul. As coleções científicas, cujo acervo é majoritariamente regional, são vinculadas a instituições federais e privadas de ensino e/ou pesquisa, e são abaixo apresentadas em ordem decrescente de número de registros de répteis: (1) Coleção Zoológica de Referência da Universidade Federal de Mato Grosso do Sul (ZUFMS). É a maior e mais representativa 
coleção científica com mais de 5.308 exemplares de anfíbios e 1.936 de répteis, majoritariamente do MS. Essa coleção é a mais importante do estado do MS, contando com o acervo incorporado do Prof. Arlindo de Figueiredo Béda (CHAFB), um dos autores desse artigo, oriundo do campus de Aquidauana. Ainda, aloca o material da Coleção Zoológica de Referência do Campus de Corumbá, Seção de Herpetologia (CEUCH), cuja numeração permanece a original, com 6.146 exemplares de anfíbios e répteis, sendo 2.115 de répteis com representantes de 131 espécies, onde 81\% (1.714) dos registros são procedentes do MS. A coleção ZUFMS está temporariamente alojada no Laboratório de Zoologia, com infraestrutura precária. Entretanto, está no aguardo da execução de projeto institucional aprovado para a construção de infraestrutura física adequada. Conta com dois técnicos de laboratório para auxílio nas atividades de curadoria. (2) Coleção de Vertebrados do Pantanal (HCPAP), Embrapa Pantanal. Conta com 413 exemplares de répteis oriundos da região da Nhecolândia e arredores, Corumbá, MS. A coleção foi recentemente reativada e retomada por projeto institucional e conta com boa infraestrutura. (3) Coleção Herpetológica do Biotério da Universidade Católica Dom Bosco (BIOTH). A coleção é recente e conta com pouco mais de uma centena de registros.

Principais lacunas de conhecimento. A fauna de répteis na maior parte do MS é pouco ou minimamente conhecida, sendo melhor inventariada, apenas, a porção centro-oeste (Fig. 1). O norte, o sul e todo o leste do estado destacam-se por apresentar escassa vegetação original, onde nenhuma área foi bem estudada. Até mesmo as UCs e RPPNs, em sua maioria, não foram inventariadas (Fig. 2). Essa porção está comprometida pelo intensivo uso da terra (ALMEIDA \& Kudlavicz, 2011), e não há sequer uma área considerada bem amostrada, o que sugere perda de biodiversidade sem ao menos conhecê-la.

Bastante preocupante é o fato de que praticamente todo o estado apresenta lacunas de amostragem de répteis. Mesmo inventários em regiões com elevada importância biológica, como o complexo Aporé-Sucuriú, no Cerrado, e ambientes aquáticos do Pantanal (incluindo a região de cabeceiras no Cerrado), são considerados insuficientemente amostrados (STRÜSSMANN et al., 2000; UETANABARO et al., 2006). Adicionalmente, cerca de $80 \%$ dos registros do MS localizados na bacia do rio Paraná são oriundos de relatórios de impactos ambientais decorrentes da implantação de grandes empreendimentos. Ainda, apenas 1,27\% da extensão territorial do estado encontra-se protegida por UCs de proteção integral e $11,42 \%$, por UCs de uso sustentável (Parques, APA, Monumento Natural e RPPNs) (WWFBRASIL, 2011). Mesmo diante desse cenário, o presente estudo mostra que, dentre as UCs e RPPNs, somente o Parque Nacional da Serra da Bodoquena (Planalto), RPPN Estância Caiman e UC Parque do Rio Negro (ambos na planície de inundação) estão em áreas consideradas melhor amostradas.

Perspectivas de Pesquisa para o Grupo nos próximos 10 anos. As grandes lacunas de amostragem de répteis no MS evidenciadas neste estudo apontam a necessidade de ações voltadas para a complementação de informações preexistentes e investimentos em áreas ainda inexploradas cientificamente. Essas ações podem ser diretas, mediante o aporte de recursos e outros incentivos (bolsas de pesquisa e de apoio técnico, por exemplo) por parte de órgãos governamentais (universidades, ICMBio, CNPq, etc.). Ainda, é importante a implantação de normas, por parte de órgãos ambientais (ICMBio, IMASUL), para que o materialtestemunho proveniente de propostas de empreendimento que necessite de licenciamento ambiental (diagnósticos ambientais, EIA, monitoramento, resgate de fauna, etc.) seja majotariamente depositado em coleções científicas no estado do Mato Grosso do Sul.

As maiores coleções zoológicas do estado (HCPAP e ZUFMS) são relativamente recentes (cerca de 20 anos) quando comparadas com outras instituições brasileiras e têm valor inestimável. Após o incêndio no Instituto Butantan (Franco, 2012), as coleções regionais passaram a ser ainda mais valorizadas, consultadas com maior frequência e, consequentemente, mais citadas em publicações científicas. Com isso, a responsabilidade e o compromisso em manter as coleções adequadamente alocadas e organizadas aumentaram. Contudo, o empenho e investimento humano e financeiro das instituições para a manutenção, informatização e estrutura física dessas coleções ainda é precário. Recentemente, com a criação do Programa Biota-MS, a informatização e organização das coleções científicas estaduais têm sido incluídas como ações prioritárias, com o intuito de conhecer, e divulgar a biodiversidade do estado.

Menos de 20\% das espécies de répteis registradas para o MS foram oficialmente avaliadas pela IUCN (IUCN, 2012) e inexiste uma lista de espécies ameaçadas no estado. Tendo em vista as crescentes perdas de habitats naturais no MS e a presença de espécies não encontradas em outros estados brasileiros, e ainda, a reduzida extensão de ocorrência de muitas destas espécies, há necessidade de incentivos estaduais e/ou federais para implementação de oficinas para avaliar o estado de conservação e de ameaça da fauna do estado.

Considerando a velocidade do uso e ocupação da terra nas regiões de cabeceiras dos rios que abastecem a planície de inundação e o escasso conhecimento quanto à fauna em geral nestas regiões e na porção norte do MS, parece prudente e necessário considerar as mesmas como áreas prioritárias para a realização de inventários de fauna e para a criação de UCs. Nas áreas apontadas como mais bem conhecidas, estudos ecológicos e evolutivos podem mostrar as necessidades específicas de conservação e potencialidades para o uso sustentável.

Agradecimentos. À Fundação de Apoio ao Desenvolvimento do Ensino, Ciências e Tecnologia do Estado de Mato Grosso do Sul (Fundect) e à Superintendência de Ciências e Tecnologia do Estado de Mato Grosso do Sul (Sucitec/MS), pelo convite de participação neste fascículo especial da Iheringia, Série Zoologia pelo suporte financeiro para sua publicação. Aos curadores das coleções Júlio Cesar de Moura-Leite (MHNCI), Gustavo Graciolli (ZUFMS), Francisco Luís Franco (IBSP), Guarino R. Colli (CHUNB), Marcos A. Carvalho (UFMT), Hussam Zaher (MZUSP) que providenciaram as listas do material e/ou permitiram o acesso ao material testemunho. À Cyntia C. Santos, Masao Uetanabaro, Paula H. Valdujo, 
Domingos J. Rodrigues, Walfrido Tomas e Ellen Wang que gentilmente cederam informações de campo. À Ana C. Lima, Júlio C. Moura-Leite, Sérgio Morato e Renato Bérnils pelo auxílio e esclarecimentos, ao Vinícius Xavier da Silva pelo auxílio na identificação de Bothrops do grupo neuwiedi, ao Valdir Germano e Daniela Gennari pela identificação de Liotyphlops beui, à Tami Mott pela identificação das espécies de Amphisbaena. Ao Reuber Brandão pela confirmação de registros depositados na CHUNB. Ao José Luiz Massao M. Sugai pelo auxílio na confecção dos mapas. À Marcelle A. Tomas (EMBRAPA-CPAP) e Kamila C. Mecchi (UCDB) pelo auxílio junto a HCPAP e BIOTH, Tainá F. Dorado-Rodrigues pelo auxílio com registros de bibliografias. Ao Instituto Homem Pantaneiro, Embrapa-Pantanal, RPPN Fazenda Alegria, RPPN Fazenda Rio Negro, Recanto Ecológico Rio da Prata, Base de Estudos do Pantanal (UFMS), Fazenda São Cristóvão e Fazenda São Bento pela autorização e/ou apoio logístico. VLF agradece às instituições de fomento FUNDECT (122/06; 329/08), CNPq (486039/20064; 307360/2009-1; 480414/2009-2; 563232/2010-2; 563352/2010-8), CPP, EMBRAPA-CPAP (02.10.06.007.00.00), PROPP-UFMS pelo apoio a projetos de pesquisa que originaram o banco de dados. RAKR agradece à CAPES pelo apoio financeiro. CS e FLS são bolsistas produtividade CNPq (Processos 309541/2012-3, 301071/2011-0, respectivamente)

\section{REFERÊNCIAS BIBLIOGRÁFICAS}

Ав'SABER, A. N. 1988. O Pantanal Mato-grossense e a teoria dos refúgios. Revista Brasileira Geografia 50:9-57.

ADÁmoli, J. A. 1982. O Pantanal e suas relações fitogeográficas com os cerrados. Discussão sobre o conceito "Complexo Pantanal". In: Congresso Nacional de Botânica. Teresina, Sociedade Brasileira de Botânica, p.109-119.

Albugueroue, N. R. \& Lema, T. 2012. Description of the second known specimen of Apostolepis intermedia (Serpentes, Colubridae, Xenodontinae). Zootaxa 3325:53-58.

Alho, C. J. R.; Strüssmann, C. \& VASCOnCellos, L. A. S. 2001. Indicadores da magnitude da diversidade e abundância de vertebrados silvestres do Pantanal num mosaico de hábitats sazonais. In: III Simpósio sobre Recursos Naturais e Socioeconômicos do Pantanal (SIMPAN), Corumbá, Embrapa (CD ROM).

Almeida, R. A. 2011. Aliança terra-capital em Mato Grosso do Sul: redefinições no campo e na cidade. In: Silva, E. A. \& De AlmeidA, R. A. org. Território e territorialidades em Mato Grosso do Sul. São Paulo, Expressão Popular. vol. 1, p.103-134.

Almeida, R. A. \& Kudlavicz, M. 2011. A potencialidade da pequena unidade de produção em Mato Grosso do Sul: Os censos agropecuários 1995/96 e 2006 em database. In: FARIAS, M. F. L. \& FAISTING, A. L. org. Direitos Humanos, diversidade e movimentos sociais: um diálogo necessário. Dourados, Editora da UFGD, p.45-66.

Arruda, L. A. G.; Ávila, R. W.; Morais, D. H. \& Kawashita-Ribeiro, R. A. 2011. Rhachidelus brazili Boulenger, 1908 (Squamata: Serpentes: Dipsadidae): New records, range extension and distribution map in Mato Grosso and Mato Grosso do Sul states, Brazil. Check List 7(6):854-855.

Assine, M. L. 2003. Sedimentação na Bacia do Pantanal Matogrossense, Centro-Oeste do Brasil. Tese de livre docência. Rio Claro, São Paulo, Universidade Estadual de São Paulo.

Barreto, D. S.; Valadẽo, R. M.; Nogueira, C.; Castro, C. P.; Ferreira, V. L. \& Strüssmann, C. 2012. New locality records, geographical distribution, and morphological variation in Cercosaura parkeri (Ruibal, 1952) (Squamata: Gymnophthalmidae) from western Brazil. Check List. 8(6): 1365-1369.

BÉRNILS, R. S. \& COSTA, H. C. 2012. Répteis brasileiros: lista de espécies. Versão 2012.2. Sociedade Brasileira de Herpetologia. Disponível em: $<$ http://www.sbherpetologia.org.br/>. Acessado em 15.02.2013.

BIVAND, R. S. \& RunDEL, C. 2012. Rgeos. Interface to Geometry Engine - Open Source (GEOS). R package version 0.2-1. Disponível em: $<$ http://www.inside-r.org/packages/rgeos $>$.

Brasil, Ministério do Meio Ambiente dos Recursos Hídricos e da Amazônia Legal (Brasília, DF). 1997. Plano de Conservação da Bacia do Alto Paraguai (Pantanal) - PCBAP. Análise integrada e prognóstico da Bacia do Alto Paraguai. Brasília, MMA. 369p.

CABrera, M. R. 2012. A new species of Cnemidophorus (Squamata, Teiidae) from the South American Chaco. Herpetological Journal 22:123-131.

Colli, G. R. 2005. As origens e a diversificação da herpetofauna do Cerrado.
In: SCARIOT, J.; SouZA-Silva, C. \& Felfili, J. M. ed. Cerrado: Ecologia, Biodiversidade e Conservação. Ministério do Meio Ambiente, Brasília, p.247-264.

Colli, G. R.; Bastos, R. P. \& AraúJo, A. F. B. 2002. The character and dynamics of the Cerrado herpetofauna. In: OlIVEIRA, P. S. \& MARQUIS, R. J. ed. The Cerrados of Brazil: Ecology and Natural History of a Neotropical Savanna. New York, Columbia University Press, p.223-241

Cressie, N. A. C. 1991. Statistics for spatial data. New York, J. Wiley \& Sons, 920p.

COSTA, H. C. \& BéRnILS, R. S. 2014. Répteis brasileiros: Lista de espécies. Herpetologia Brasileira. 3(3): 74-84. Disponível em: <http://www. sbherpetologia.org.br/>. Acessado em 24.08.2015.

Ferrão, M. 2011. Serpentes da Fazenda Nhumirim, Pantanal da Nhecolândia, Mato Grosso do Sul, Brasil: Composição e comparações com outras taxocenoses sulamericanas. Dissertação de Mestrado. Cuiabá, Universidade Federal de Mato Grosso.

Ferreira, V. L.; Petroni, D. M. \& CÁrCeres, N. C. 2005. Bachia bresslaui. Geographic distribution. Herpetological Review 36(1): 78 .

FranCo, F. L. 2012. A Coleção Herpetológica do Instituto Butantan: da sua origem ao incêndio ocorrido em 15 de maio de 2010. Herpetologia Brasileira 1(1):22-31. Disponível em: <http://www.sbherpetologia. org.br/herpbras/2012-1.html>. Acessado em 09.10.2012.

FundaÇ̃̃o CÂNDIDo Rondon. 2007. Diagnóstico Ecosocioambiental de Bonito Mato Grosso do Sul. Relatório Final. Disponível em: $<\mathrm{http}: / /$ www.fcr.org.br>. Acessado em 1512.2012.

Hamilton, S. K; Sippel, S. \& Melack, J. 1996. Inundation patterns in the Pantanal wetland of South America determined from passive microwave remote sensing. Archiv für Hydrobiologie 137(3):1-23.

Hammer, O.; Harper, D. A. T. \& Ryan, P. D. 2001. PAST: Paleontological Statistics Software Package for Education and Data Analysis. Paleontologia Eletronica 4(1):1-9. Disponível em: $<\mathrm{http}: / /$ palaeoelectronica.org/2001_1/past/issue1_01.htm>.

Harris, M. B.; Arcangelo, C.; Pinto, E. C. T.; Camargo, G.; Ramos Neto, M. B. \& Silva, S. M. 2005a. Estimativas de perda da área natural da Bacia do Alto Paraguai e Pantanal Brasileiro. Campo Grande, Conservação Internacional . Relatório técnico não publicado. Disponível em: $<$ http://www.conservation.org.br/arquivos/Perda-dearea-natural-daBAP.pdf $>$.

Harris, M. B.; Tomas, W. M.; Mourão, G.; Silva, C. J.; Guimarães, E.; Sonoda, F. \& FACHim, E. 2005b. Desafios para proteger o Pantanal brasileiro: ameaças e iniciativas em conservação. Megadiversidade 1:156-164

Hijmans, R. J.; Guarino, L.; Cruz, M. \& Rojas, E. 2001. Computer tools for spatial analysis of plant genetic resources data: 1. DIVA-GIS. Plant Genetic Resources Newsletter 127:15-19.

IBGE - Instituto Brasileiro de Geografia e Estatística. 2004. Mapa de Biomas do Brasil, primeira aproximação. Rio de Janeiro, IBGE. Disponível em: $<$ http://www.ibge.gov.br/home/geociencias/default prod.shtm>.

IBGE - Instituto Brasileiro de Geografia e Estatística. 2011. Mapa de Biomas do Brasil. Disponível em: <ftp://ftp.ibge.gov.br/Cartas_e Mapas/Mapas_Murais/>.

iCMBio - Instituto Chico Mendes de Conservação da Biodiversidade. 2015. Lista de espécies ameaçadas. Disponível em $<\mathrm{http} / / \mathrm{www}$. icmbio.gov.br/portal/biodiversidade/fauna-brasileira/lista-de-especies. html > . Acessado em 27.08.2015

IUCN. Red List of Threatened Species. Version 2012. Disponível em: $<$ www.iucnredlist.org $>$. Acessado em 1201.2013.

Junk, W. J.; Cunha, C. N.; Wantzen, K. M.; Petermann, P.; Strüssmann, C.; MARQues, M. I. \& AdIS, J. 2006. Biodiversity and its conservation in the Pantanal of Mato Grosso, Brazil. Aquatic Sciences 68:278-309.

KAWASHITA-RIBEIRO, R. A. 2007. História natural de uma taxocenose de serpentes da RPPN Acurizal e áreas adjacentes, Serra do Amolar, borda oeste do Pantanal, Corumbá, Mato Grosso do Sul, Brasil. Dissertação de mestrado. Cuiabá, Universidade Federal de Mato Grosso.

Klink, C. A. \& MoreirA, A. G. 2002. Past and current human occupation, and land use. In: Oliveira, P. S. \& Marquis, R. J. org. The Cerrados of Brazil Ecology and Natural History of a Neotropical Savanna. New York, Columbia University Press, p.69-90. 
Landgref Filho, P. \& Aoki, C. 2011. Helicops infrataeniatus (Water Snake). Herpetological Review 42:394.

Lewin-Koh, N. J.; Bivand, R.; Pebesma, E. J.; Archer, E. \& Baddeley, A. 2009. Maptools. Tools for reading and handling spatial objects. $\mathrm{R}$ package version 0.7-26. Disponível em: $<\mathrm{http}: / / C R A N . R-p r o j e c t$. org/package=maptools $>$. Acessado em 15.11.2012.

Lourival, R.; Harris, M. \& Montambault, J. R. 2000. Introdução ao Pantanal, Mato Grosso do Sul, Brasil. In: Willink, P. W.; Chernoff, B.; Alonso, L. E.; Montambault, J. R. \& Lourival, R. ed. A biological assessment of the aquatic ecosystems of the Pantanal, Mato Grosso do Sul, Brasil. Washington, Conservation International. (RAP Bulletin of Biological Assessment, n.18), p.146-151.

MMA - Ministério do MeIo AMBIENTE. 2002. Biodiversidade brasileira. Avaliação e identificação de áreas e ações prioritárias para conservação, utilização sustentável e repartição de benefícios da biodiversidade brasileira. Brasília, MMA/SBF. 404p.

MMA - Ministério do Meio Ambiente. 2003. Lista das espécies da fauna brasileira ameaçadas de extinção. Brasília, MMA. (Instrução Normativa $\mathrm{n}^{\circ} 3$ ). $19 \mathrm{p}$.

MMA - Ministério do Meio Ambiente. 2007a. Biodiversidade do Cerrado e Pantanal: áreas e ações prioritárias para conservação da biodiversidade. Brasília, MMA/SBF. 540p.

MMA - Ministério do Meio Ambiente. 2007b. Mapa das Áreas Prioritárias para conservação, uso sustentável e repartição de benefícios da biodiversidade brasileira. Disponível em: $<\mathrm{http}: / /$ www.mma.gov.br>. Acessado em 25.09.2012.

Nogueira, C.; Ribeiro, S.; Costa, G. C. \& Colli, G. R. 2011. Vicariance and endemism in a Neotropical savanna hotspot: distribution patterns of Cerrado squamate reptiles. Journal of Biogeography 38:1907-1922.

Pereira, D. N.; Stender-Oliveira, F.; Rodrigues, M. G. \& Bérnils, R. S. 2007. Distribution and habitat use of Sordellina punctata (Serpentes, Colubridae), with a new record from State of São Paulo, Brasil. Herpetological Bulletin 100:18-22.

PIAU, E. S. 2012. Diagnóstico espacial da expansão da cana-de-açúcar e sua relação indireta com a atividade da pecuária nos estados de São Paulo, Goiás e Mato Grosso do Sul. In: Encontro Nacional da Anppas. Belém, Anppas, p. 1-18.

PorZECANSKI, A. L. \& CRACRAFT, J. 2005. Cladistic analysis of distributions and endemism (CADE): using raw distributions of birds to unravel the biogeography of the South American aridlands. Journal of Biogeography 32:261-275.

Potт, A. \& AdÁmoli, J. 1999. Unidades de vegetação do Pantanal dos Paiaguás. In: II Simpósio sobre Recursos Naturais e Socioeconômicos do Pantanal SIMPAN. Corumbá, Embrapa, p.183-202.

Prado, D. E.; GibBs, P. E.; Pott, A. \& Pott, V. J. 1992. The Chaco-Pantanal transition in southern Mato Grosso, Brazil. In: FurLey, P. A.; Proctor, J. \& RAtTeR, J. A. ed. Nature and dynamics of forest-savanna boundaries. London, Chapman and Hall, p.451-470.

Prance, G. T. \& Schaller, G. B. 1982. Preliminary study of some vegetation types of the Pantanal, Mato Grosso, Brazil. Brittonia 34(2):228-251.

R CORe TeAm. 2012. R A language and environment for statistical computing. Vienna, R Foundation for Statistical Computing. Disponível em: <http://www.R-project.org/>. Acessado em 10.01.2013.

RBMA - Reserva da Biosfera da Mata Atlântica. 2007. Reserva da Biosfera do Pantanal. Disponível em: $<$ http://www.rbma.org.br/mab/ unesco $03 \mathrm{rb}$ pantanal.asp>. Acessado em 21.01.2013.

Rocha, C. F. D.; Bergallo, H. G.; Pombal JR., J. P.; Geise, L.; Van-Sluys, M.; Fernandes, R. \& CaRAMaschi, U. 2004. Fauna de anfíbios, répteis e mamíferos do estado do Rio de Janeiro, sudeste do Brasil. Publicações Avulsas do Museu Nacional 104:3-23.

Silva JR, N. J.; Cintra, C. E. D.; Silva, H. L. R.; Costa, M. C.; Souza, C. A.; PACHÊCo-JR., A. A. \& GonÇALVES, F. A. 2009. Herpetofauna, Ponte de Pedra hydroelectric power plant, states of Mato Grosso and Mato Grosso do Sul, Brazil. Check List 5(3):518-525.

Souza, F. L.; Uetanabaro, M.; Langref-Filho, P.; Piatti, L. \& Prado, C. P. A. 2010. Herpetofauna, Municipality of Porto Murtinho, Chaco region, Mato Grosso do Sul State, Brazil. Check List 6(3):470-475.
Strüssmann, C.; Prado, C. P. A.; Uetanabaro, M. \& Ferreira, V. L. 2000. Amphibians and Reptiles survey of select lies in the southern Pantanal floodplains and surrounding Cerrado, Mato Grosso do Sul, Pantanal. Cap. 8, Appendices 7-12 (Levantamento de anfíbios e répteis de localidades selecionadas na porção sul da planície alagável do Pantanal e Cerrado do entorno, Mato Grosso do Sul, Brasil, Apêndices 7 a 12). In: Willink, P. W.; Chernoff, B.; Alonso, L. E.; Montambault, J. R. \& Lourival, R. ed. A Biological Assessment of the Aquatic Ecosystems of the Pantanal, Mato Grosso do Sul, Brasil. Washington, Conservation International, p. 98-102, 219-223.

Strüssmann, C.; Kawashita-Ribeiro, R. A.; Ferreira, V. L. \& Beda, A. F. 2007. Herpetofauna do Pantanal Brasileiro. In: NASCimento L. B. \& Oliveira, M. E. org. Herpetologia no Brasil II. Belo Horizonte, Sociedade Brasileira de Herpetologia, p.66-84.

Strüssmann, C.; Prado, C. P. A.; Ferreira, V. L. \& Kawashita-Ribeiro, R. A. 2011. Diversity, ecology, management and conservation of amphibians and reptiles of the Brazilian Pantanal: a review. In: JUNK, W. J.; Da Silva, C. J.; Cunha, C. N. \& Wantzen, K. M. org. The Pantanal. Ecology, biodiversity and sustainable management of a large neotropical seasonal wetland. Sophia, Pensoft, p. 497-522.

Sugai, J. L. M. M.; Souza, F. L.; Uetanabaro, M. \& Landgref Filho, P. L. 2012. Cnemidophorus jalapensis (Whiptail lizard). Herpetological Review 43:444.

Swartz, F. A. 2000. The Pantanal. Undestanding and preserving the world's largest wetland. St. Paul, Paragon House. 301p.

Thomas, R. A.; Bérnils, R. S.; Moura-Leite, J. C. \& Morato, S. A. A. 2006. Redescription of Ditaxodon taeniatus (Hensel, 1868) (Serpentes, Colubridae, Xenodontinae): variation, relationships, and distribution. South American Journal of Herpetology 1(2):94-101.

Uetanabaro, M.; Guimarães, L. D.; BéDa, A. F.; Landgref Filho, P.; Prado, C. P. A.; Bastos, R. P. \& Ávila, R. W. 2006. Inventário da herpetofauna do Complexo Jauru. In: PAGotTo, T. C. S. \& SouZA, P. R. org. Biodiversidade do Complexo Aporé-Sucuriú: subsídios à conservação e ao manejo do Cerrado. Campo Grande, Editora UFMS, p.103-112.

Uetanabaro, M.; Souza, L. F.; Landgref, P.; Beda, A. F. \& Brandão, R. A. 2007. Anfíbios e répteis da Serra da Bodoquena, Mato Grosso do sul, Brasil. Biota Neotropica 7(3):279-289.

Vanzolini, P. E. 1963. Problemas faunísticos do Cerrado. In: Simpósio Sobre o Cerrado. São Paulo, Editora da Universidade de São Paulo, p.307-321.

Wang, E.; Ferreira, V. L. \& Himmelstein, J. 2004. Amphibians and reptiles of the Southern Pantanal. In: Chandler, M. \& WANG, E. ed. The Pantanal Conservation Research Initiative. Maynard, Annual Report Earthwatch Institute, p.34-44.

Wang, E.; Ferreira, V. L.; Himmelstein, J. \& Strüssmann, C. 2005. Amphibians and reptiles of the Southern Pantanal. In: CHANDLER, M.; Wang, E. \& Johansson, P. ed. The Pantanal Conservation Research Initiative. Maynard, Annual Report Earthwatch Institute, p.30-38.

WerNeCK, F. P. 2011. The diversification of eastern South American open vegetation biomes: Historical biogeography and perspectives. Quaternary Science Reviews 30:1630-1648.

WWF Brasil - World Wildlife Fund Brasil. 2011. Efetividade de gestão das unidades de conservação no estado do Mato Grosso do Sul. Mato Grosso do Sul, WWF-Brasil, Secretaria de Estado de Meio Ambiente, do Planejamento, da Ciência e Tecnologia (Semac), Instituto de Meio Ambiente do Mato Grosso do Sul. Disponível em: $<$ http://www.wwf.org.br/informacoes/noticias meio ambiente e natureza/?30683/Publicao-avalia-unidades-de-conservao-do-MS $>$. Acessado em 19.01.2013.

Zaher, H.; Barbo, F. E.; Martinez, P. S.; Nogueira, C.; Rodrigues, M. T. \& SAWAYA, R. J. 2011. Répteis do estado de São Paulo: conhecimento atual e perspectivas. Biota Neotropica 11(1a):1-15. Disponível em: $<$ http://www.biotaneotropica.org.br/v11n1a/pt/abstract?inventory+b n0051101a201>

ZANELLA, F. C. 2002. Systematics and biogeography of the bee genus Caenonomada Asmead, 1899 (Hymenoptera: Apidae: Tapinotaspidini). Studies on Neotropical Fauna and Environment 37:249-261. 